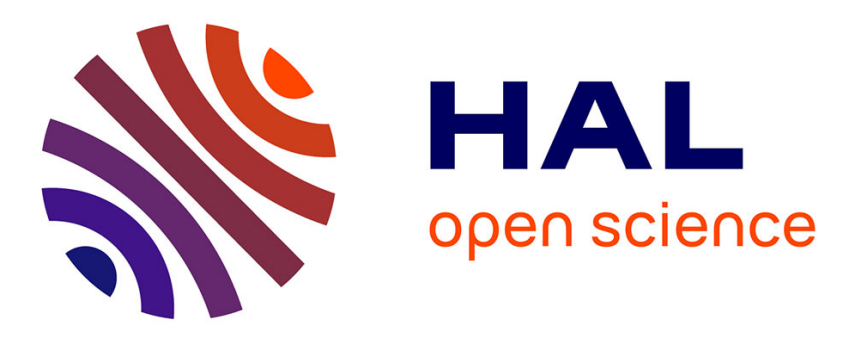

\title{
Effective behavior of thermo-elastic tubes with wavy layers
}

Dimitrios Tsalis, Georges Chatzigeorgiou, Nicolas Charalambakis

\section{To cite this version:}

Dimitrios Tsalis, Georges Chatzigeorgiou, Nicolas Charalambakis. Effective behavior of thermoelastic tubes with wavy layers. Composites Part B: Engineering, 2016, 99, pp.173-187. 10.1016/j.compositesb.2016.05.043 . hal-01364573

\section{HAL Id: hal-01364573 https://hal.science/hal-01364573}

Submitted on 12 Sep 2016

HAL is a multi-disciplinary open access archive for the deposit and dissemination of scientific research documents, whether they are published or not. The documents may come from teaching and research institutions in France or abroad, or from public or private research centers.
L'archive ouverte pluridisciplinaire HAL, est destinée au dépôt et à la diffusion de documents scientifiques de niveau recherche, publiés ou non, émanant des établissements d'enseignement et de recherche français ou étrangers, des laboratoires publics ou privés. 


\title{
Effective behavior of thermo-elastic tubes with wavy layers
}

\author{
Dimitrios Tsalis ${ }^{\mathrm{a}}$, George Chatzigeorgiou ${ }^{\mathrm{b}}$, Nicolas Charalambakis ${ }^{\mathrm{a}}$ \\ ${ }^{a}$ Department of Civil Engineering, Aristotle University of Thessaloniki,GR 54124, \\ Thessaloniki, Greece \\ ${ }^{b}$ LEM3-UMR 7239 CNRS, Arts et Metiers ParisTech Metz-Lorraine, 4 Rue Augustin \\ Fresnel 57078 Metz, France
}

\begin{abstract}
In the present article, the homogenization of a composite star-shaped tube with numerous thin, periodic, elastic wavy layers, is presented. The composite exhibits a multiscale periodicity allowing for a multistep asymptotic homogenization scheme starting from the finest scale. The scheme gives the complete effective thermoelastic behavior of the composite. By a numerical example of a two-phase composite with sinusoidal wavy walls, whose effective behavior is an orhotropic material, the above method is illustrated.
\end{abstract}

Key words: A. Multi-scale shell; B. Wavy walls; C. Periodic homogenization.

\section{Introduction}

Shell composites are the subject of intense research effort recently (see Guinovart-Sanjuan et al. (2016) for a general methodology of homogenization and a nice short survey on elastic laminate shell composites). Previously, Popovich and Fedai (1997) presented the axisymmetric problem of thermoelasticity of a multilayer thermosensitive tube. Tarn and Wang (2001) studied 
the mechanical response of laminated composite tubes. In nanomechanics, Seidel (2007) proved the multifunctional nature of carbon-nanotube-polymer nanocomposites. Chatzigeorgiou et al. (2008) studied the homogenization of elastic hollow cylinders with discontinuous properties (see also Tsukrov and Drach (2010) for composites with cylindrically orthotropic layers and Nie and Batra (2010) for material tailoring of functionally graded hollow circular cylinders). Cavalcante et al. (2009) investigated the transient thermomechanical analysis of a layered cylinder by the parametric finite-volume theory. Multifunctional shell structures present desirable electronic, magnetic, optical or thermal properties, combined with each other or with stiffness, ductility and strengthening. The multifuctionality of multiscale composites can be enhanced by building the material from the smaller to the greater scale mimicking the nature structures (Salonikitis et al. (2010)). Chatzigeorgiou et al. (2011) presented the homogenization of aligned fuzzy fiber composites. Seidel et al. (2014) studied the case of fuzzy fibers based on multi-walled carbon nanotubes. Many bio-inspired systems present shell structure needing further investigation.

In a parallel way, stratified structures with wavy layers occuring accidentaly or deliberately, attracted the attention of many researchers due to their waviness-induced strengthening under specific straining path (see for instance Khatam and Pindera (2012), Tu and Pindera (2013), targeting the finite deformation response of porcine mitral valve strut chordae based on a simplified wavy model). Waviness of layers may occur accidentally during the manufacturing process in thin metallic and ceramic multilayers for applications in magnetic, optoelectronic and high-speed electronics technology or 
may deliberately be induced to enhance selected properties (Khatam and Pindera (2009b)). Composites with wavy multilayer architecture were proposed for experimentally usage as isolation system in the space laser anti-missile program by NASA. They showed high inherent damping and stiffness while preserving the light weight advantages of composites (Pratt et al. (2000)). The tube with wavy wall is one more example of a structure attempting to replicate natural structures: complex biomolecular fluids are transported along a membrane composed of wavy-rough nanoannuli (Chu (2013)).

Khatam and Pindera investigated the effective behavior of elastic wavy periodic multilayers for the first time using micromechanics based homogenization. In Khatam and Pindera (2009b), thermoelastic moduli of periodic multilayers with wavy architectures were computed. Additionally, Khatam and Pindera (2009a) revealed the important role that plasticity plays on the post-yield regime relative to the corresponding flat configuration. Moreover, concerning the same regime, in Khatam and Pindera (2010) the elastic layer thickness is shown to have a substantial effect depending on waviness and loading mode. It is known that fiber wavy architectures may occur during fabrication of thin fiber-reinforced composite laminates with bad consequences on compressive strength (Adams and Bell (1995), Kugler and Moon (2002)). Taylor and Steigmann (2009) simulated thermoelastic membranes and studied wrinkling and slackening effects of laminated thin films. Recently, wavy architectures have been used in nanotechnology to enhance certain strength properties, or to reduce thermal warpage in printed circuit boards (Grenestedt and Hutapea (2002)). Wavy interfacial morphologies during manufacturing process in nanotechnology may affect the overall be- 
havior of the composite. Rough multi-walls can be modeled by wavy layers. Minicomposites made via chemical vapor infiltration reinforced with fibers are convenient specimens for interface evaluation (see Sauder et al. (2008)). Nano- and micro elements are the basis of primary complex structures in the form of multilayer nanoshell tubes made of alternating layers of metal and polymers (Barmak and Coffey (2014)). Wavy fibers were studied in Kundalwal and Ray (2014); Ray and Kundalwal (2013, 2014).

The effective thermal conductivity of composites is seriously affected by a possible thermal barrier resistance at the interface between the constituents or by the high thermal conductivity of thermal interfacial materials. Manual processing may result in serious roughness of interfaces. Thermal stability of multilayered structures (in the form of layers with an adhesive layer between) improve heat dissipation of light-emitting diodes. Graphene reinforced SiBCN ceramics exhibit thermal shock resistance superior to monoliths of the same material. Also toughening mechanisms including pull-out and bridging appear to improve thermal shock response (Li et al. (2016)). In multilayered-structured ceramic coating design for thermal barrier coatings, dense layers lead to large thermal conductivity and stiffness but small thermal life time, needing optimization of the process through the introduction of lamellae well-bonded dense layer between the conventional porous materials and the superalloy bond coat (Liu et al. (2016a)). Joining of ceramic matrix composites $(\mathrm{Cf} / \mathrm{SiC})$ to high temperature ceramics ( $\mathrm{SiC}$ multilayers) improves thermal protection of aerospace heat protection systems (Jimenez et al. (2016)).

In Tsalis et al. (2012) and Tsalis et al. (2013) the elastic and plastic 
homogenization of layered wavy composites is studied using a generalized periodicity-based semi-analytical method. In the context of biomimicry, Katz et al. (2015) studied biomimetic materials with material architectures that resemble wavy brick-and-mortar structures found in nacre, using finite volume direct averaging micromechanics theory and proved that waviness induces increase in ductility. While flat architectures are accessible to analytical solutions, wavy architectures need a combination of cell problems and computational techniques. Specifically for multi-scale materials with structural hierarchy (Lakes (2005)), a succession of cell problems and, possibly, micromechanics methods at specific scales where the corresponding layers are composites with finer scale, may be necessary (see Tsalis et al. (2015). One-dimensional cell problems are obtained only by applying a generalized periodicity-based homogenization scheme, while micromechanics techniques as the Mori-Tanaka method (Qu and Cherkaoui (2006)) are used only in the interior of composite layers exhibiting two-dimensional periodicity.

The intent of the present paper is to combine the two above geometries, i.e the shell geometry with the wavy architecture, by considering shells made of wavy cylindrical layers exhibiting two-dimensional periodicity thus needing a FEM implementation in the framework of cartesian coordinates. In Liu et al. (2016b) the axial dynamic performance of thin-walled tubes with starshaped cross section is studied. In the present paper, one of the desired goals is to contribute to the effort for tailoring the elastic and thermal behavior of the above tubes under different strain paths. The proposed homogenization method is based on the assumption that different periodicity scales coexist in the tube, i.e. a very fine scale in the interior of every layer, a fine scale in the 
radial direction and a sufficiently coarse scale in the angular direction. This allows one to apply multi-scale homogenization techniques in three steps starting from the finest scale. The second and the third steps lead to an one-dimensional cell problem which has analytical solutions and gives closed forms for the effective stiffness coefficients. The homogenization in these two steps is based on the concept of generalized periodicity presented in Tsalis et al. (2012) (see also Guinovart-Sanjuan et al. (2016) for a more general methodology of shell structures) and follows the schema of the step-by-step homogenization in Tsalis et al. (2015). The method proposed in this paper reduces to the known results for strictly circular or non-wavy composites or laminated tubes as a way of validation.

The paper is organized as follows: in Section 2 we pose the problem with all needed assumptions on the periodicity scales, we find the equations of the cell problem and we present the analytical solution for the unknown gradients of the displacement fluctuation. Moreover, we give the analytical expressions for the effective stiffness, thermal expansion and conductivity coefficients. In Section 3, we present a numerical example of a two-phase tube made of alternative stiff and soft isotropic materials with different thermal coefficients and obtain its effective (orthotropic) behavior. Strengthening and weakening effects are analyzed, correlated to certain straining cases. Finally, in Section 4, we present some concluding remarks. 


\section{A multiscale homogenization scheme}

2.1. Statement of the problem, periodicity assumptions and outline of the method

In the sequel, we adopt the description of multiscale periodic functions proposed in classical homogenization (Bensoussan et al. (1978), Briane (1990), Briane (1993)): the heterogeneous material under consideration is characterized by heterogeneity parameters $\left\{\epsilon_{1}, \epsilon_{2}, \epsilon_{3} \ldots\right\}$, denoting a set of periodicity scales which are assumed to be of different order (see Milton (2002), for the analysis of sequentially layered composites). A function $\varphi$ exhibiting periodicity $\epsilon$ with respect to a material surface $\varrho$ is written formally $\varphi^{\epsilon}(x):=\varphi\left(x, \frac{\varrho}{\varepsilon}\right)$. In cylindrical coordinates $x=(r, \theta, z)$, a function exhibiting periodicities $\epsilon^{m}, \epsilon^{n}, \epsilon^{l}$ with respect to $\varrho_{1}, \varrho_{2}, \varrho_{3}$ respectively is writ-

ten $\varphi^{\epsilon}(r, \theta, z):=\varphi\left(r, \theta, z, \frac{\varrho_{1}}{\varepsilon^{m}}, \frac{\varrho_{2}}{\varepsilon^{n}}, \frac{\varrho_{3}}{\varepsilon^{l}}\right)$. We use the operators (Chatzigeorgiou et al. (2012))

$$
\begin{gathered}
L_{r}=\frac{\partial}{\partial r}, L_{\theta}=\frac{1}{r} \frac{\partial}{\partial \theta}, L_{z}=\frac{\partial}{\partial z}, \\
P_{1}=\nabla_{r} \varrho, P_{2}=\frac{1}{r} \nabla_{\theta} \varrho
\end{gathered}
$$

to write the equilibrium equations of a heterogeneous material

$$
\begin{gathered}
L_{j} \sigma_{r j}^{\epsilon}+\frac{\sigma_{r r}^{\epsilon}-\sigma_{\theta \theta}^{\epsilon}}{\stackrel{r}{\sigma}_{\epsilon}^{\epsilon}}+f_{r}=0, \\
L_{j} \sigma_{\theta j}^{\epsilon}+2 \frac{{ }_{r \theta}^{r}}{r}+f_{\theta}=0, \\
L_{j} \sigma_{z j}^{\epsilon}+\frac{\sigma_{r z}^{\epsilon}}{r}+f_{z}=0,
\end{gathered}
$$

the constitutive equations

$$
\sigma_{i j}^{\epsilon}=C_{i j k l}^{\epsilon}\left[\varepsilon_{k l}^{\epsilon}-\alpha_{k l}^{\epsilon}\left(T^{\epsilon}-T_{r e f}\right)\right]
$$


the strain-displacement relations

$$
\begin{gathered}
\varepsilon_{r r}^{\epsilon}=L_{r} u_{r}^{\epsilon}, \varepsilon_{\theta \theta}^{\epsilon}=L_{\theta} u_{\theta}^{\epsilon}+\frac{u_{r}^{\epsilon}}{r}, \varepsilon_{z z}^{\epsilon}=L_{z} u_{z}^{\epsilon}, \\
\varepsilon_{\theta z}^{\epsilon}=\frac{1}{2}\left(L_{\theta} u_{z}^{\epsilon}+L_{z} u_{\theta}^{\epsilon}\right), \varepsilon_{r z}^{\epsilon}=\frac{1}{2}\left(L_{r} u_{z}^{\epsilon}+L_{z} u_{r}^{\epsilon}\right) \\
\varepsilon_{r \theta}^{\epsilon}=\frac{1}{2}\left(L_{r} u_{\theta}^{\epsilon}+L_{\theta} u_{r}^{\epsilon}\right),
\end{gathered}
$$

and the energy equation for steady state heat flow and negligible elastic mechanical energy

$$
\begin{gathered}
L_{i} q_{i}^{\epsilon}+\frac{q_{r}}{r}=Q, \\
q_{k}=-k_{k j}^{\epsilon} L_{j} T^{\epsilon}, k=r, \theta, z .
\end{gathered}
$$

In the above equations, $u_{i}$ denotes the displacement vector, $\sigma_{i j}$ the stress tensor, $\varepsilon_{i j}$ the strain tensor, $C_{i j k l}$ the fourth-order elasticity tensor, $T$ the temperature, $Q$ the radiation, $q$ the heat flux, $\alpha_{i j}$ the coefficients of thermal expansion tensor, $k_{i j}$ the coefficients of thermal conductivity tensor, where all free latin indices take the values $r, \theta, z$, while repetition of only the latin indices means summation over $r, \theta, z$. The above system must be supplemented by Neumann or Dirichlet or mixed boundary conditions in order to be solved. However, in the asymptotic expansion homogenization (AEH) method, the boundary conditions do not affect the effective properties of the composite, if it is made of elastic constituents only.

We consider a star-shaped tube made of numerous thin wavy layers. Cylindrical wavy multilayers may be comprised of alternating soft and stiff homogeneous phases (see Khatam and Pindera (2009b) for stratified wavy structures), or of a metal matrix reinforced by continuous fibers in arbitrary orientations, resulting to transversely isotropic or monoclinic behavior inside each individual layer, or of a polymeric matrix (Salzar et al. (1996)). 


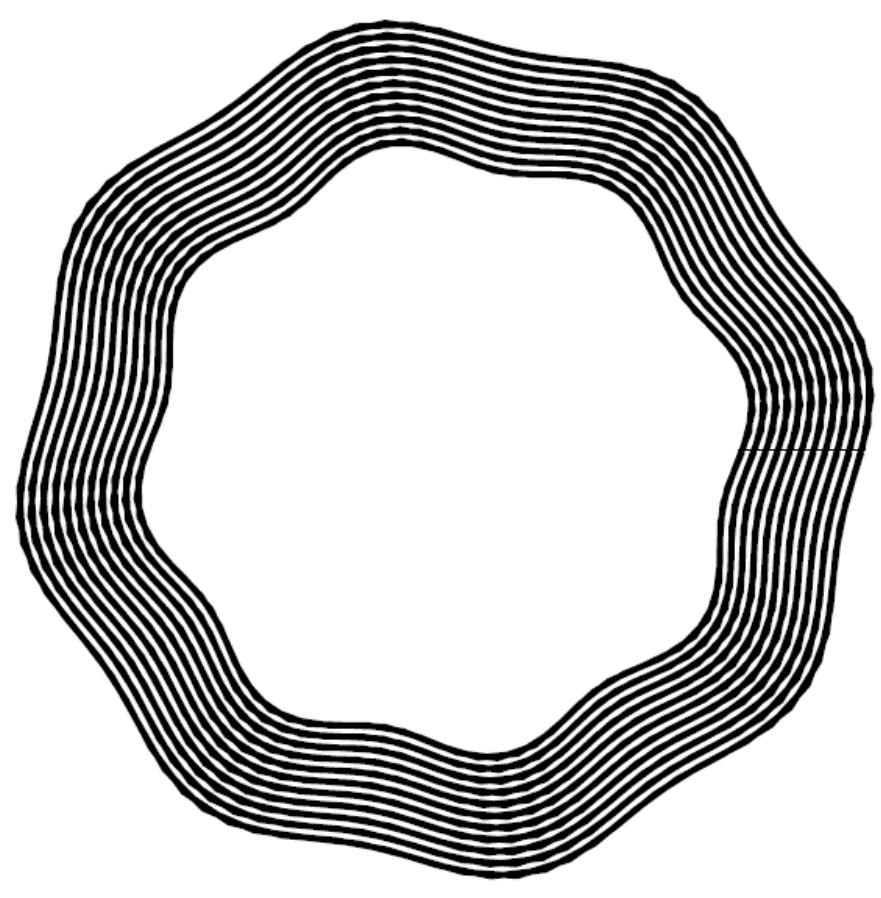

Figure 1: Cross section of the tube with wavy walls vertical to its axes.

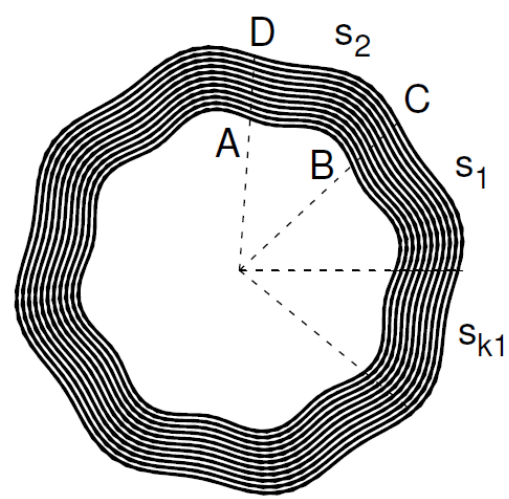

(a)

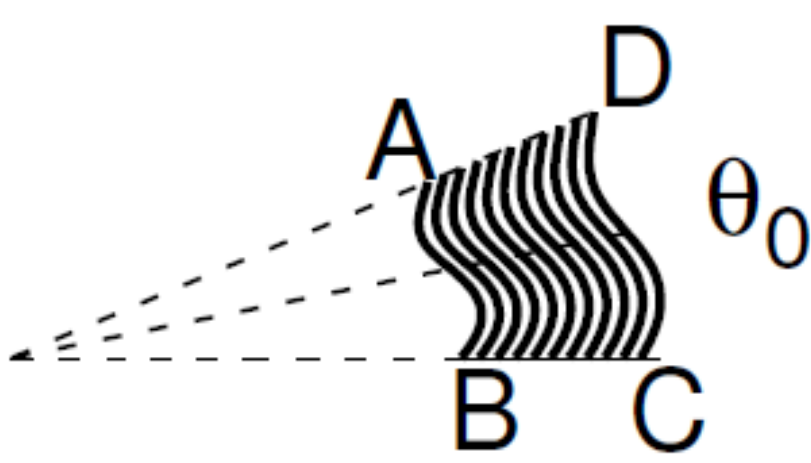

(b)

Figure 2: (a) Cross section of the tube divided into $k_{1}$ sectors and (b) a typical sector. 
In the present problem, it could be of interest to study the impact of wavy layers or wavy interlayer morphologies to the overall mechanical or thermomechanical behavior of the tube assuming that the wavy layers exhibit a double periodicity with respect to material surfaces $\varrho(r, \theta)=$ const very close between them, forming a microstructure (see fig. 1). All thermomechanical functions are assumed to depend on the macro-coordinates $(r, \theta)$ and additionally to be periodic with respect to the material surfaces $\varrho(r, \theta)=$ const. An example of such a periodic microstructure is the microstructure defined by the parametric equations of sinusoidal wavy layered walls

$$
\begin{gathered}
\varrho_{1}=r+H \sin \left(k_{1} \theta\right)=k \epsilon, \quad k=1,2 \ldots, \\
\varrho_{2}=\theta=k \epsilon^{m}, \quad m<\frac{1}{2}, \quad k=1,2 \ldots
\end{gathered}
$$

In fig.2 the structure is divided into $k_{1}$ sectors of typical form ABCD. Along the $\varrho_{1}$-direction, the layers present a periodicity $\epsilon$ of the order $\frac{t}{k_{2}}$, where $t$ the thickness of the tube and $k_{2}$ the number of the layers. The thickness $t$ is assumed to be small relatively to the inner radius of the tube. The angular periodicity is assumed to be of the order $\epsilon^{m}, m<\frac{1}{2}$. We note that it is possible that each layer is heterogeneous with a periodic structure. In this case, the periodicity is assumed to be of the order $\epsilon^{2}$. For the notion of the generalized periodicity function the reader is invited to consult Tsalis et al. $(2012,2013)$. We proceed to a multi-step homogenization based on the above assumption, i.e. that the periodicity with respect to the $\varrho_{1}$-direction is much finer than the angular periodicity and that the heterogeneities inside every layer are much finer than the thickness of the layers. The first step consists in homogenizing the layers by a periodic homogenization FEM or 
micromechanics based method. We focus to the next two steps. The second step (called $1^{\text {st }}$ in the sequel) consists in performing the homogenization with respect to the $\varrho_{1}$-direction thus obtaining a periodically, in the angular direction, graded structure. In the final step (called $2^{\text {nd }}$ in the sequel), the structure is homogenized with respect to the angular direction.

\subsection{First step of homogenization}

In the sequel, the (possible) homogenization in the interior of the layers is omitted and the structure, to be further homogenized, possesses homogeneous layered walls. As far as the $1^{\text {st }}$ step of homogenization is concerned, we consider that, in the sector ABCD of fig.2 and for a "mean" angle $\theta_{0}$, all functions exhibit periodicity only with respect to $\varrho_{1}$ in the first equation of (2.6), denoted in the sequel for simplicity by $\varrho$. Then, every function is expressed as

$$
\varphi^{\epsilon}=\varphi\left(r, \theta_{0}, \frac{\varrho}{\epsilon}\right)=\varphi\left(r, \theta_{0}, \bar{\varrho}\right)
$$

with periodicity with respect to $\bar{\varrho}$, where

$$
\bar{\varrho}=\frac{\varrho}{\epsilon}
$$

In the sequel, we simplify the above expression by writing

$$
\varphi^{\epsilon}=\varphi\left(r, \theta, \frac{\varrho}{\epsilon}\right)=\varphi(r, \theta, \bar{\varrho}) .
$$

Then the gradient of every function becomes

$$
\begin{aligned}
& \nabla_{r} \varphi^{\epsilon}=\nabla_{r} \varphi+\frac{1}{\epsilon} \nabla_{r} \varrho \nabla_{\varrho} \varphi, \\
& \nabla_{\theta} \varphi^{\epsilon}=\nabla_{\theta} \varphi+\frac{1}{\epsilon} \nabla_{\theta} \varrho \nabla_{\bar{\varrho}} \varphi .
\end{aligned}
$$


Following the AEH method, all functions are written in a series expansion form

$$
\varphi^{\epsilon}=\varphi^{(0)}(r, \theta, \bar{\varrho})+\epsilon \varphi^{(1)}(r, \theta, \bar{\varrho})+\epsilon^{2} \varphi^{(2)}(r, \theta, \bar{\varrho})+\ldots
$$

where $\varphi^{(0)}, \varphi^{(1)}, \varphi^{(2)} \ldots$ are assumed to be periodic with respect to $\bar{\varrho}$.

By defining the operators in polar coordinates (Chatzigeorgiou et al. (2011))

$$
L_{r}^{(1)}=\frac{\partial}{\partial r}=\nabla_{r}, L_{\theta}^{(1)}=\frac{1}{r} \frac{\partial}{\partial \theta}=\frac{1}{r} \nabla_{\theta}, L_{z}^{(1)}=\frac{\partial}{\partial z}=\nabla_{z},
$$

and

$$
\bar{L}_{r}^{(1)}=P_{1}^{(1)} \frac{\partial}{\partial \varrho}, \bar{L}_{\theta}^{(1)}=P_{2}^{(1)} \frac{\partial}{\partial \varrho}, \bar{L}_{z}^{(1)}=0,
$$

all operators $L_{i}^{(1)}, i=r, \theta, z$, are substituted by $L_{i}^{(1)}+\frac{1}{\epsilon} \bar{L}_{i}^{(1)}$. In addition, we remind that $P_{1}^{(1)}=\nabla_{r} \varrho$ and $P_{2}^{(1)}=\frac{1}{r} \nabla_{\theta} \varrho$.

Using the expanded forms of displacement, strain, stress and heat flux and replacing in the equilibrium and in the energy equations, one obtains from the condition of zero coefficients of $\epsilon^{-2}$ and $\epsilon^{-1}$ that the mechanical cell problem reads (Chatzigeorgiou et al. (2012))

$$
\begin{gathered}
\bar{L}_{j}^{(1)} C_{i j m n}+\bar{L}_{j}^{(1)}\left(C_{i j k l} \bar{L}_{k}^{(1)} N_{l}^{m n(1)}\right)=0, \\
\bar{L}_{j}^{(1)} C_{i j k l} a_{k l}+\bar{L}_{j}^{(1)} C_{i j k l} \bar{L}_{k}^{(1)} N_{l}^{0(1)}=0,
\end{gathered}
$$

where $N_{i}^{m n(1)}, N_{i}^{0(1)}$ unknown functions, periodic in $\varrho$ satisfying the continuity equations 


$$
\begin{aligned}
& {\left[\left|N_{i}^{m n(1)}\right|\right]=0, \quad\left[\left|\left(C_{i j m n}+C_{i j k l} \bar{L}_{k}^{(1)} N_{l}^{m n(1)}\right) n_{j}\right|\right]=0, \quad i, m, n=r, \theta, z,} \\
& {\left[\left|N_{i}^{0(1)}\right|\right]=0, \quad\left[\left|\left(C_{i j k l} a_{k l}+\left(C_{i j k l} \bar{L}_{k}^{(1)} N_{l}^{0(1)}\right)\right) n_{j}\right|\right]=0, \quad i=r, \theta, z, \quad}
\end{aligned}
$$

while the thermal cell problem reads (Chatzigeorgiou et al. (2012))

$$
\bar{L}_{i}^{(1)} \kappa_{i m}+\bar{L}_{i}^{(1)} \kappa_{i j} \bar{L}_{j}^{(1)} W_{m}^{(1)}=0,
$$

where $W_{i}^{(1)}$ unknown functions periodic in $\varrho$ satisfying the continuity equations

$$
\left[\left|W_{m}^{(1)}\right|\right]=0, \quad\left[\left|\left(\kappa_{i m}+\kappa_{i j} \bar{L}_{j}^{(1)} W_{m}^{(1)}\right) n_{i}\right|\right]=0, \quad m=r, \theta, z .
$$

We note that the above system of equations (2.15), (2.16), (2.19) under the continuity conditions (2.17), (2.18), (2.20) and periodicity conditions can be solved with respect to the derivatives $\bar{L}_{i}^{(1)} N_{j}^{m n}{ }^{(1)}, \bar{L}_{i}^{(1)} N_{j}^{0}{ }^{(1)}, \bar{L}_{i}^{(1)} W_{m}^{(1)}$. The full expansion of equations (2.15), (2.16), (2.19) (using Voigt notation for (2.15), (2.16)) are presented in (A.1), (A.2) and (A.5) respectively of Appendix A.

The solution of the cell problem (2.15) is of the form 


$$
\begin{gathered}
\frac{\partial N_{r}^{a}(1)}{\partial \bar{\varrho}}=\frac{\mathrm{B}_{2}^{(1)}\left(\lambda_{1}^{a(1)}-P_{1}^{(1)} C_{6 a}-P_{2}^{(1)} C_{2 a}\right)-\mathrm{A}_{2}^{(1)}\left(\lambda_{2}^{a(1)}-P_{1}^{(1)} C_{1 a}-P_{2}^{(1)} C_{2 a}\right)}{\mathrm{A}_{1}^{(1)} \mathrm{B}_{2}^{(1)}-\mathrm{A}_{2}^{(1)} \mathrm{B}_{1}^{(1)}} \\
\frac{\partial N_{\theta}^{a(1)}}{\partial \bar{\varrho}}=\frac{\mathrm{A}_{1}^{(1)}\left(\lambda_{2}^{a(1)}-P_{1}^{(1)} C_{1 a}-P_{2}^{(1)} C_{2 a}\right)-\mathrm{B}_{1}^{(1)}\left(\lambda_{1}^{a(1)}-P_{1}^{(1)} C_{6 a}-P_{2}^{(1)} C_{2 a}\right)}{\mathrm{A}_{1}^{(1)} \mathrm{B}_{2}^{(1)}-\mathrm{A}_{2}^{(1)} \mathrm{B}_{1}^{(1)}} \\
\frac{\partial N_{z}^{a(1)}}{\partial \bar{\varrho}}=\frac{\lambda_{3}^{a(1)}-P_{1}^{(1)} C_{5 a}-P_{2}^{(1)} C_{4 a}}{P_{1}^{(1)} C_{55} P_{1}^{(1)}+P_{2}^{(1)} C_{44} P_{2}^{(1)}}
\end{gathered}
$$

$\alpha=1,2,3,4,5,6$, where the coefficients $\mathrm{A}_{1}^{(1)}, \mathrm{A}_{2}^{(1)}, \mathrm{B}_{1}^{(1)}, \mathrm{B}_{2}^{(1)}, \lambda_{1}^{\alpha(1)}, \lambda_{2}^{\alpha(1)}, \lambda_{3}^{\alpha(1)}$ are given in (B.15) and (B.16) of Appendix B.

The solution of the cell problem (2.16) is of the form

$$
\begin{gathered}
\frac{\partial N_{r}^{0}{ }^{(1)}}{\partial \varrho}=\frac{B_{2}^{(1)}\left(\bar{\lambda}_{1}^{a(1)}-A_{3}^{(1)}\right)-A_{2}^{(1)}\left(\bar{\lambda}_{2}^{a(1)}-B_{3}^{(1)}\right)}{A_{1}^{(1)} B_{2}^{(1)}-A_{2}^{(1)} B_{1}^{(1)}} \\
\frac{\partial N_{\theta}^{0}(1)}{\partial \bar{\varrho}}=\frac{A_{1}^{(1)}\left(\bar{\lambda}_{2}^{a(1)}-B_{3}^{(1)}\right)-B_{1}^{(1)}\left(\bar{\lambda}_{1}^{a}(1)-A_{3}^{(1)}\right)}{A_{1}^{(1)} B_{2}^{(1)}-A_{2}^{(1)} B_{1}^{(1)}} \\
\frac{\partial N_{z}^{0}(1)}{\partial \bar{\varrho}}=\frac{\bar{\lambda}_{3}^{a(1)}-D_{2}^{(1)}}{D_{1}^{(1)}}
\end{gathered}
$$

where the coefficients $A^{(1)}, B^{(1)}, D^{(1)}$ are given in (A.4) of Appendix A and $\bar{\lambda}_{1}^{a(1)}, \bar{\lambda}_{2}^{a(1)}, \bar{\lambda}_{3}^{a(1)}$ are given in (B.19) of Appendix B.

Finally, the solution of the thermal cell problem (2.19) is of the form 


$$
\begin{aligned}
& \frac{\partial W_{r}^{(1)}}{\partial \bar{\varrho}}=\frac{\tilde{\lambda}_{1}^{a(1)}-F_{1}^{(1)}}{F_{0}^{(1)}}, \\
& \frac{\partial W_{\theta}^{(1)}}{\partial \bar{\varrho}}=\frac{\tilde{\lambda}_{2}^{a(1)}-F_{2}^{(1)}}{F_{0}^{(1)}}, \\
& \frac{\partial W_{z}^{(1)}}{\partial \bar{\varrho}}=\frac{\tilde{\lambda}_{3}^{a(1)}-F_{3}^{(1)}}{F_{0}^{(1)}},
\end{aligned}
$$

where the coefficients $F_{0}^{(1)}, F_{1}^{(1)}, F_{2}^{(1)}, F_{3}^{(1)}$ are given in (A.7) of Appendix A and $\tilde{\lambda}_{1}^{a(1)}, \tilde{\lambda}_{2}^{a(1)}, \tilde{\lambda}_{3}^{a(1)}$ are given in (B.21) of Appendix B.

The above expressions for $\bar{L}_{i}^{(1)} N_{j}^{m n}{ }^{(1)}, \bar{L}_{i}^{(1)} N_{j}^{0}{ }^{(1)}, \bar{L}_{i}^{(1)} W_{m}^{(1)}$ are needed for the homogenized coefficients resulting from the $1^{\text {st }}$ step of homogenization

$$
\begin{gathered}
C_{b a}^{e f f(1)}=<C_{b a}+C_{b 1} \bar{L}_{r}^{(1)} N_{r}^{a}+C_{b 6} \bar{L}_{r}^{(1)} N_{\theta}^{a}+C_{b 5} \bar{L}_{r}^{(1)} N_{z}^{a}+ \\
+C_{b 6} \bar{L}_{\theta}^{(1)} N_{r}^{a}+C_{b 2} \bar{L}_{\theta}^{(1)} N_{\theta}^{a}+C_{b 4} \bar{L}_{\theta}^{(1)} N_{z}^{a}> \\
a_{b}^{e f f(1)}=\left(C_{b a}^{e f f(1)}\right)^{-1}<C_{a 1} \alpha_{1}+C_{a 2} \alpha_{2}+C_{a 3} \alpha_{3}+\left(C_{a 1} \bar{L}_{r}^{(1)}+C_{a 6} \bar{L}_{\theta}^{(1)}\right) N_{r}^{0}{ }^{(1)}+ \\
+\left(C_{a 6} \bar{L}_{r}^{(1)}+C_{a 2} \bar{L}_{\theta}^{(2)}\right) N_{\theta}^{0(2)}+\left(C_{a 5} \bar{L}_{r}^{(1)}+C_{a 4} \bar{L}_{\theta}^{(2)}\right) N_{z}^{0(3)}>,
\end{gathered}
$$

or

$$
\begin{aligned}
a_{b}^{e f f(1)}=\left(C_{b a}^{e f f(1)}\right)^{-1}< & C_{a 1} \alpha_{1}+C_{a 2} \alpha_{2}+C_{a 3} \alpha_{3}+\left(C_{11} \alpha_{1}+C_{12} \alpha_{2}+C_{13} \alpha_{3}\right) \bar{L}_{r}^{(1)} N_{r}^{a(1)}+ \\
& +\left(C_{21} \alpha_{1}+C_{22} \alpha_{2}+C_{23} \alpha_{3}\right) \bar{L}_{\theta}^{(1)} N_{\theta}^{a(1)}>
\end{aligned}
$$

for $a, b=1,2,3,4,5,6$ and

$$
\kappa_{i m}^{e f f(1)}=<\kappa_{i m}+\kappa_{i r} \bar{L}_{r}^{(1)} W_{r}^{(1)}+\kappa_{i \theta} \bar{L}_{r}^{(1)} W_{\theta}^{(1)}>,
$$

for $i, m=r, \theta, z$, where the operator $<\ldots>$ denotes the mean value. The homogenized material corresponds to a periodically graded material. These coefficients will be used as input data for the second step of homogenization. 


\subsection{Second step of homogenization}

The next step of homogenization ( $2^{\text {nd }}$ step) is performed with respect to the angular direction and leads again to an one-dimensional cell problem. The heterogeneous anisotropic material under consideration admits as elastic coefficients the coefficients obtained from the previous step. Using again the same notations, where for simplicity now $\varrho$ is used instead of $\varrho_{2}$, we obtain the expressions

$$
\begin{gathered}
\varphi^{\epsilon}=\varphi\left(\theta, \frac{\theta}{\epsilon}\right)=\varphi(\theta, \bar{\theta}), \\
\nabla_{r} \varphi^{\epsilon}=0, \\
\nabla_{\theta} \varphi^{\epsilon}=\nabla_{\theta} \varphi+\frac{1}{\epsilon} \nabla_{\theta} \varrho \nabla_{\bar{\theta}} \varphi \\
L_{r}^{(2)}=\frac{\partial}{\partial r}=\nabla_{r}, L_{\theta}^{(2)}=\frac{1}{r} \frac{\partial}{\partial \theta}=\frac{1}{r} \nabla_{\theta}, L_{z}^{(2)}=\frac{\partial}{\partial z}=\nabla_{z}, \\
\bar{L}_{r}^{(2)}=0, \bar{L}_{\theta}^{(2)}=P_{2}^{(2)} \frac{\partial}{\partial \bar{\theta}}, \bar{L}_{z}^{(2)}=0 .
\end{gathered}
$$

In addition we remind that, $P_{1}^{(2)}=\nabla_{r} \varrho$ and $P_{2}^{(2)}=\frac{1}{r} \nabla_{\theta} \varrho$.

The cell problems have now the following form

$$
\bar{L}_{\theta}^{(2)} C_{i \theta m n}^{e f f(1)}+\bar{L}_{\theta}^{(2)}\left(C_{i \theta k l}^{(e f f 1)} \bar{L}_{k}^{(2)} N_{l}^{m n(2)}\right)=0,
$$

for $i, m, n=r, \theta, z$ (no summation on $\theta)$,

$$
\bar{L}_{j}^{(2)} C_{i j k l}^{e f f(1)} a_{k l}^{e f f(1)}+\bar{L}_{j}^{(2)} C_{i j k l}^{e f f(1)} \bar{L}_{k}^{(2)} N_{l}^{0(2)}=0,
$$

where $N_{i}^{m n(2)}, N_{i}^{0(2)}$ unknown functions, periodic in $\bar{\theta}$ satisfying the continuity equations 


$$
\begin{aligned}
& {\left[\left|N_{i}^{m n(2)}\right|\right]=0, \quad\left[\left|\left(C_{i j m n}^{e f f(1)}+C_{i j k l}^{e f f}{ }^{(1)} \bar{L}_{k}^{(2)} N_{l}^{m n(2)}\right) n_{j}\right|\right]=0, \quad i, m, n=r, \theta, z,} \\
& (2.35) \\
& {\left[\left|N_{i}^{0(2)}\right|\right]=0, \quad\left[\left|\left(C_{i j k l}^{e f f(1)} a_{k l}^{e f f(1)}+\left(C_{i j k l}^{e f f(1)} \bar{L}_{k}^{(2)} N_{l}^{0(2)}\right)\right) n_{j}\right|\right]=0, \quad i=r, \theta, z,}
\end{aligned}
$$

while the thermal cell problem reads (Chatzigeorgiou et al. (2012))

$$
\bar{L}_{i}^{(2)} \kappa_{i m}^{e f f(1)}+\bar{L}_{i}^{(2)} \kappa_{i j}^{e f f(1)} \bar{L}_{j}^{(2)} W_{m}^{(2)}=0,
$$

where $W_{i}^{(2)}$ unknown functions periodic in $\bar{\theta}$ satisfying the continuity equations

$$
\left[\left|W_{m}^{(2)}\right|\right]=0, \quad\left[\left|\left(\kappa_{i m}^{e f f(1)}+\kappa_{i j}^{e f f}{ }^{(1)} \bar{L}_{j}^{(2)} W_{m}^{(2)}\right) n_{i}\right|\right]=0, \quad m=r, \theta, z .
$$

The full expansion of (2.33), (2.34) (using Voigt notation) and (2.37) is presented in (A.8), (A.9) and (A.12) respectively in Appendix A.

The solution of the cell problem (2.33) is of the form

$$
\begin{aligned}
& \frac{\partial N_{r}^{a(2)}}{\partial \bar{\theta}}=\frac{\left(\lambda_{1}^{a(2)}-P_{2}^{(2)} C_{2 \alpha}^{e f f(1)}\right) C_{22}^{e f f(1)}-\left(\lambda_{2}^{a(2)}-P_{2}^{(2)} C_{6 \alpha}^{e f f(1)}\right) C_{62}^{e f f(1)}}{P_{2}^{2}{ }^{(2)}\left(C_{26}^{e f f}{ }^{(1)} C_{62}^{e f f(1)}-C_{66}^{e f f(1)} C_{22}^{e f f(1)}\right)}, \\
& \frac{\partial N_{\theta}^{a(2)}}{\partial \bar{\theta}}=\frac{-\left(\lambda_{1}^{a(2)}-P_{2}^{(2)} C_{6 \alpha}^{e f f(1)}\right) C_{26}^{e f f(1)}+\left(\lambda_{2}^{a(2)}-P_{2}^{(2)} C_{2 \alpha}^{e f f(1)}\right) C_{66}^{e f f(1)}}{P_{2}^{2(2)}\left(C_{26}^{e f f(1)} C_{62}^{e f f(1)}-C_{66}^{e f f(1)} C_{22}^{e f f(1)}\right)} \\
& \frac{\partial N_{z}^{a(2)}}{\partial \bar{\theta}}=\frac{\lambda_{3}^{a(2)}-P_{2}^{(2)} C_{4 \alpha}^{e f f(1)}}{P_{2}^{2(2)} C_{44}^{e f f(1)}}
\end{aligned}
$$


where the coefficients $\lambda_{1}^{a(2)}, \lambda_{2}^{a(2)}, \lambda_{3}^{a(2)}$ are given in (B.22) of Appendix B.

The solution of the cell problem (2.34) is of the form

$$
\begin{gathered}
\frac{\partial N_{r}^{0}{ }^{(2)}}{\partial \bar{\theta}}=\frac{B_{2}^{(2)}\left(\bar{\lambda}_{1}^{a(2)}-A_{3}^{(2)}\right)-A_{2}^{(2)}\left(\bar{\lambda}_{2}^{a(2)}-B_{3}^{(2)}\right)}{A_{1}^{(2)} B_{2}^{(2)}-A_{2}^{(2)} B_{1}^{(2)}} \\
\frac{\partial N_{\theta}^{0}{ }^{(2)}}{\partial \bar{\theta}}=\frac{A_{1}^{(2)}\left(\bar{\lambda}_{2}^{a(2)}-B_{3}^{(2)}\right)-B_{1}^{(2)}\left(\bar{\lambda}_{1}^{a(2)}-A_{3}^{(2)}\right)}{A_{1}^{(2)} B_{2}^{(2)}-A_{2}^{(2)} B_{1}^{(2)}} \\
\frac{\partial N_{z}^{0}{ }^{(2)}}{\partial \bar{\theta}}=\frac{\bar{\lambda}_{3}^{a(2)}-D_{2}^{(2)}}{D_{1}^{(2)}}
\end{gathered}
$$

where the coefficients $\bar{\lambda}_{1}^{a(2)}, \bar{\lambda}_{2}^{a(2)}, \bar{\lambda}_{3}^{a(2)}$ are given in (B.23) of Appendix B.

The solution of the cell problem (2.37) is of the form

$$
\begin{aligned}
& \frac{\partial W_{r}^{(2)}}{\partial \bar{\theta}}=\frac{\tilde{\lambda}_{1}^{a(2)}-P_{2}^{(2)} \kappa_{\theta r}^{e f f(1)}}{P_{2}^{(2)} \kappa_{\theta \theta}^{e f f(1)} P_{2}^{(2)}}, \\
& \frac{\partial W_{\theta}^{(2)}}{\partial \bar{\theta}}=\frac{\tilde{\lambda}_{2}^{a(2)}-P_{2}^{(2)} \kappa_{\theta \theta}^{e f f(1)}}{P_{2}^{(2)} \kappa_{\theta \theta}^{e f f(1)} P_{2}^{(2)}}, \\
& \frac{\partial W_{z}^{(2)}}{\partial \bar{\theta}}=\frac{\tilde{\lambda}_{3}^{a(2)}-P_{2}^{(2)} \kappa_{\theta z}^{e f f(1)}}{P_{2}^{(2)} \kappa_{\theta \theta}^{e f f(1)} P_{2}^{(2)}},
\end{aligned}
$$

where the coefficients $\tilde{\lambda}_{1}^{a(2)}, \tilde{\lambda}_{2}^{a(2)}, \tilde{\lambda}_{3}^{a(2)}$ are given in (B.25) of Appendix B.

\subsection{Effective properties}

Using the elastic and thermal coefficients obtained from the $1^{\text {st }}$ step of homogenization and the values of $\frac{\partial N_{i}^{a(2)}}{\partial \bar{\theta}}, \frac{\partial N_{i}^{0(2)}}{\partial \bar{\theta}}$ and $\frac{\partial W_{i}^{(2)}}{\partial \bar{\theta}}$ obtained from the $2^{\text {nd }}$ step homogenization we obtain the effective thermoelastic properties of the composite. More specifically, the analytical expressions for the effective 
elasticity tensor coefficients in Voigt notation read

$$
C_{b a}^{e f f}=<C_{b a}^{e f f(1)}+C_{b 6}^{e f f(1)} \bar{L}_{\theta}^{(2)} N_{r}^{a}+C_{b 2}^{e f f}{ }^{(1)} \bar{L}_{\theta}^{(2)} N_{\theta}^{a}+C_{b 4}^{e f f}{ }^{(1)} \bar{L}_{\theta}^{(2)} N_{z}^{a}>,
$$

for $a, b=1,2,3,4,5,6$. The above expressions correspond to an orthotropic elastic response.

The analytical expressions of the thermal expansion coefficients read

$$
\begin{aligned}
& a_{b}^{e f f}=\left(C_{b a}^{e f f}\right)^{-1}<C_{a 1}^{e f f(1)} \alpha_{1}^{e f f(1)}+C_{a 2}^{e f f(1)} \alpha_{2}^{e f f(1)}+C_{a 3}^{e f f(1)} \alpha_{3}^{e f f(1)}+ \\
& +2 C_{a 6}^{e f f(1)} \alpha_{6}^{e f f(1)}+C_{a 6}^{e f f(1)} \bar{L}_{\theta}^{(2)} N_{r}^{0(2)}+C_{a 2} \bar{L}_{\theta}^{(2)} N_{\theta}^{0(2)}+C_{a 4} \bar{L}_{\theta}^{(2)} N_{z}^{0}{ }^{(2)}>
\end{aligned}
$$

for $a, b=1,2,3,4,5,6$ or

$$
\begin{aligned}
& a_{b}^{e f f}=\left(C_{b a}^{e f f}\right)^{-1}<C_{a 1}^{e f f(1)} \alpha_{1}^{e f f(1)}+C_{a 2}^{e f f(1)} \alpha_{2}^{e f f(1)}+C_{a 3}^{e f f(1)} \alpha_{3}^{e f f(1)}+2 C_{a 6}^{e f f(1)} \alpha_{6}^{e f f(1)}+
\end{aligned}
$$

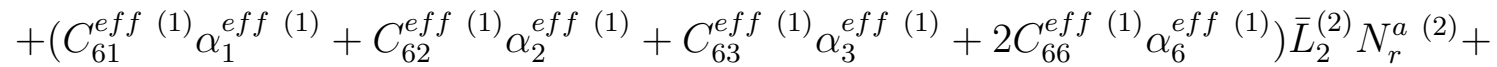

$$
\begin{aligned}
& \left(C_{21}^{e f f(1)} \alpha_{1}^{e f f(1)}+C_{22}^{e f f(1)} \alpha_{2}^{e f f(1)}+C_{23}^{e f f(1)} \alpha_{3}^{e f f(1)}+2 C_{26}^{e f f(1)} \alpha_{6}^{e f f(1)}\right) \bar{L}_{2}^{(2)} N_{\theta}^{a(2)}>
\end{aligned}
$$

for $a, b=1,2,3,4,5,6$ and $C_{i j m n}^{e f f}\left(C_{m n k l}^{e f f}\right)^{-1}=\frac{1}{2}\left(\delta_{i k} \delta_{j l}+\delta_{i l} \delta_{j k}\right)$.

Finally, the effective thermal conductivity coefficients read

$$
\kappa_{i m}^{e f f}=<\kappa_{i m}^{e f f(1)}+\kappa_{i r}^{e f f(1)} \bar{L}_{r}^{(2)} W_{m}^{(2)}+\kappa_{i \theta}^{e f f(1)} \bar{L}_{\theta}^{(2)} W_{m}^{(2)}>
$$

for $i, m=r, \theta, z$.

\section{Numerical examples. Results and discussion}

In this section, we will apply the proposed homogenization technique in order to determine the overall behavior of a star-shaped tube with multilayered sinusoidal walls with parametric equations (2.6), made of two alternative 
elastic isotropic materials, whose properties are shown in tables 1-4. For the $1^{\text {st }}$ step of homogenization,

$$
\begin{gathered}
\nabla_{r} \varrho^{(1)}=1+k_{1} H \theta \cos \left(k_{1} \theta\right), \\
\nabla_{\theta} \varrho^{(1)}=k_{1} H \cos \left(k_{1}, \theta\right),
\end{gathered}
$$

while for the $2^{\text {nd }}$ step of homogenization,

$$
\begin{aligned}
& \nabla_{r} \varrho^{(2)}=0, \\
& \nabla_{\theta} \varrho^{(2)}=1 .
\end{aligned}
$$

In fig. 3 we see the variation with respect to the angular direction of the coefficients of stiffness matrix deduced from the $1^{\text {st }}$ step of homogenization. We verify that large gradient of the wavy layers enhances shear strength in all planes (fig.3(c)) and tensile strength along the angular direction (fig.3(a)). On the other hand, it reduces the tensile strength along the radial direction. In fig. 4 we see the variation with respect to the angular direction of the thermal expansion coefficients resulting from the $1^{\text {st }}$ step. The values of the coefficients of the thermal expansion coefficient tensor vary between the respective values of the components of the composite. At points where the gradient of the layer is zero (points 4 and 10), all coefficients are equal to the corresponding coefficients in Chatzigeorgiou et al. (2012), while the effective coefficients $\alpha_{1}, \alpha_{3}$ and $\alpha_{6}$ take their maximum and $\alpha_{2}$ its minimum value. At points where the gradient of the layer takes its maximum value (points 1 , 7 and 13) the opposite holds. In addition, the effective value $\alpha_{6}$ for the $1^{\text {st }}$ step of homogenization is oscillating around zero (fig. 4(d)). 
In fig. 5 we see the variation with respect to the polar angle of the thermal conductivity coefficients resulting from the $1^{\text {st }}$ step of homogenization. Zones of the layer with large wavy gradient exhibit large radial conductivity (fig.5(a)) and small angular conductivity (fig.5(b)). Finally, concerning the effective thermal conductivity from the $1^{\text {st }}$ step of homogenization, the variation of the coefficients of the tensor in a sector is presented in fig.5, varying between the respective values of the components of the composite. At points with zero gradient (points 4 and 10), the coefficients are equal to the corresponting coefficients in Chatzigeorgiou et al. (2012). At these points the effective coefficient $\kappa_{r r}$ takes its minimum value while $\kappa_{\theta \theta}$ takes its maximum value. At points where the gradient takes its maximum value (points 1, 7 and 13) the opposite holds. In addition, the effective value $\kappa_{r \theta}$ for the $1^{s t}$ step of homogenization is oscillating around zero (fig.4(d)).

In table 5 , the effective stiffness matrix, resulting from the $2^{\text {nd }}$ step of homogenization, is shown. We verify that the overall behavior is orthotropic. From these values, we find the effective Young moduli $E_{r r}=118.778$ and $E_{\theta \theta}=116.497$ and the effective shear modulus $G_{r \theta}=23.103$. Comparison with the corresponding values of the hollow cylinder with the same geometrical and thermomechanical properties but without wavy layers (see Chatzigeorgiou et al. (2012), where $E_{r r}=118.717, E_{\theta \theta}=139.567$ and $\left.G_{r \theta}=19.978\right)$ shows an important strengthening of the shear resistance 15.6\%. On the contrary, the structure exhibits an important weakening of the hoop stress capacity $16.5 \%$. In table 6 we see the effective thermal expansion tensor. We verify that, comparing to the tube without wavy layers, the radial effective expansion coefficient is reduced $15 \%$, while the angular expansion coefficient 
is enhanced $20 \%$. Finally, in table 7 we see the effective thermal conductivity tensor. We verify that the thermal conductivity is larger in the radial direction than in the angular direction, contrarily to the wavyless case. Moreover, we verify that, comparing to the tube without wavy layers (Chatzigeorgiou et al. (2012)), the radial effective conductivity coefficient is enhanced $19 \%$, while the angular conductivity coefficient is reduced $23 \%$.

Table 1: Thermomechanical properties of constituents and volume fraction.

\begin{tabular}{|c|c|c|}
\hline Property & Material 1 (Steel) & Material 2 (Aluminum) \\
\hline \hline Young Modulus $(\mathrm{GPa})$ & 206.742 & 72.041 \\
\hline Poisson Ratio & 0.30 & 0.35 \\
\hline Thermal expansion coefficient $(1 / \mathrm{K})$ & $12.265 \mathrm{E}-6$ & $23.201 \mathrm{E}-6$ \\
\hline Coefficient of heat conductivity $(\mathrm{W} /(\mathrm{mK}))$ & 65.106 & 207.498 \\
\hline Volume fraction & $50 \%$ & $50 \%$ \\
\hline
\end{tabular}


Table 2: Symmetric stiffness tensor coefficients of the two constituents (in Voigt notation and in GPa).

\begin{tabular}{|c|c|c|}
\hline Mechanical Properties & Material 1 (Steel) & Material 2 (Aluminum) \\
\hline \hline$C_{11}, C_{22}, C_{33}$ & 278.307 & 115.621 \\
\hline$C_{12}, C_{13}, C_{23}$ & 119.274 & 62.258 \\
\hline$C_{44}, C_{55}, C_{66}$ & 79.516 & 26.682 \\
\hline$C_{14}, C_{15}, C_{16}$ & 0 & 0 \\
\hline$C_{24}, C_{25}, C_{26}$ & 0 & 0 \\
\hline$C_{34}, C_{35}, C_{36}$ & 0 & 0 \\
\hline
\end{tabular}

Table 3: Symmetric thermal expansion tensor (in $\frac{1}{K}$ ) of the two constituents.

\begin{tabular}{|c|c|c|}
\hline Properties & Material 1 (Steel) & Material 2 (Aluminum) \\
\hline \hline$\alpha_{1}, \alpha_{2}, \alpha_{3}$ & $12.265 \mathrm{E}-6$ & $23.201 \mathrm{E}-6$ \\
\hline$\alpha_{4}, \alpha_{5}, \alpha_{6}$ & 0.0 & 0.0 \\
\hline
\end{tabular}

Table 4: Symmetric thermal conductivity tensor (in $\frac{W}{m K}$ ) of the two constituents.

\begin{tabular}{|c|c|c|}
\hline Properties & Material 1 (Steel) & Material 2 (Aluminum) \\
\hline \hline$\kappa_{r r}, \kappa_{\theta \theta}, \kappa_{z z}$ & 65.106 & 207.498 \\
\hline$\kappa_{r \theta}, \kappa_{r z}, \kappa_{z \theta}$ & 0.0 & 0.0 \\
\hline
\end{tabular}




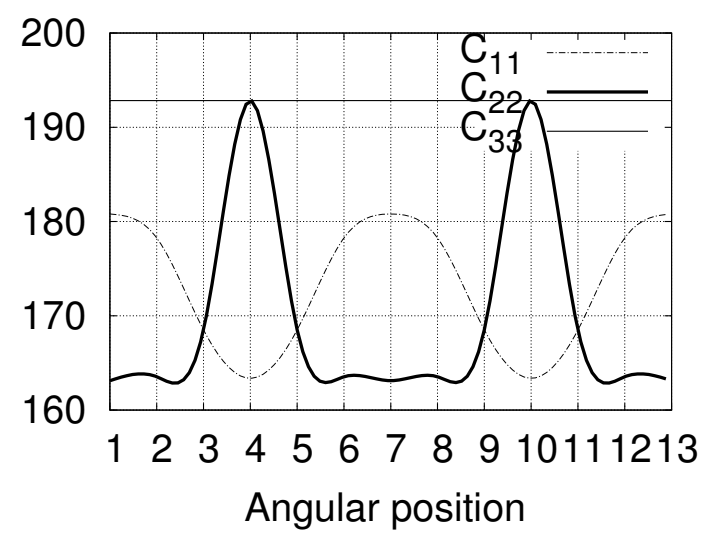

(a)

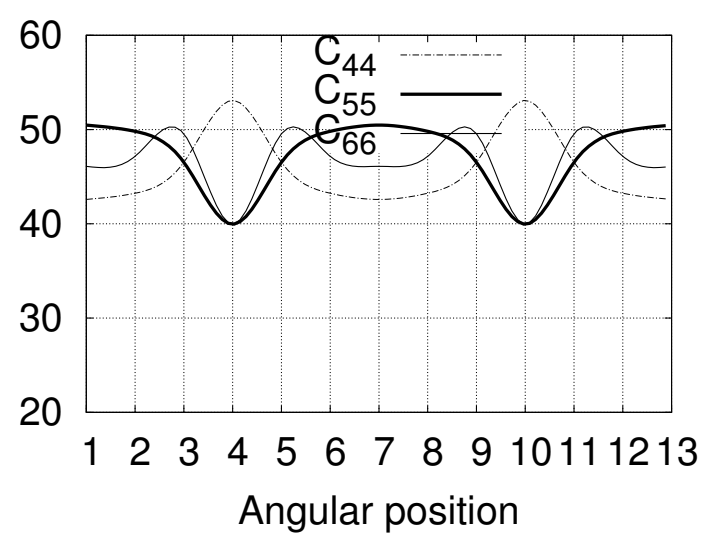

(c)

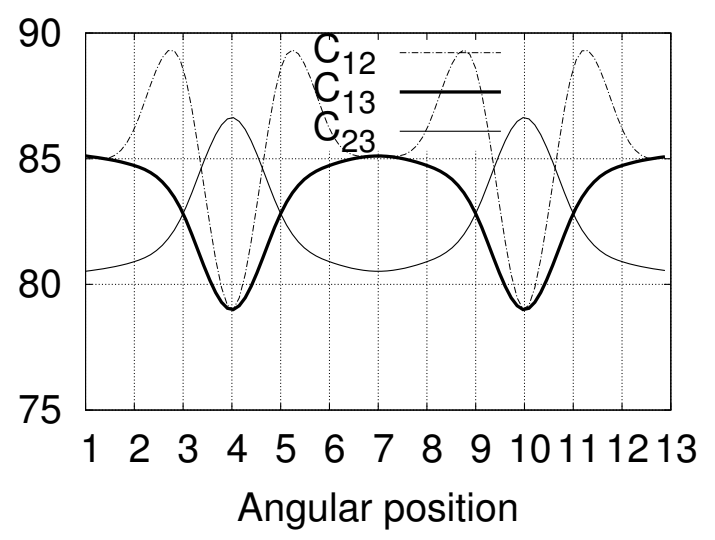

(b)

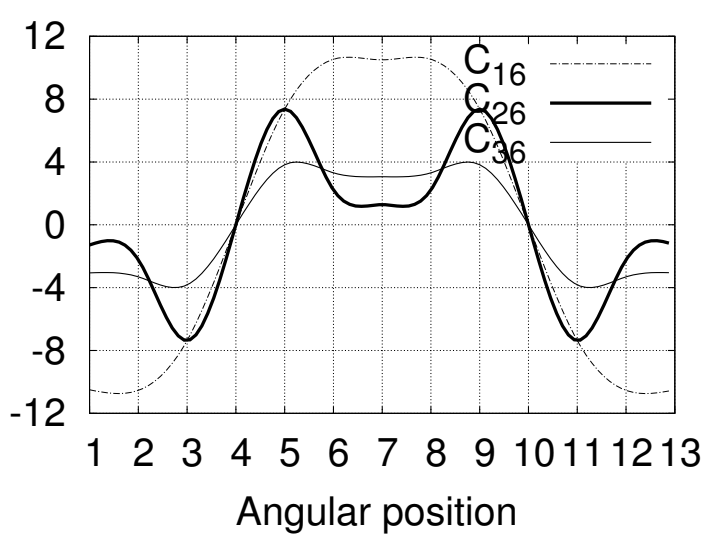

(d)

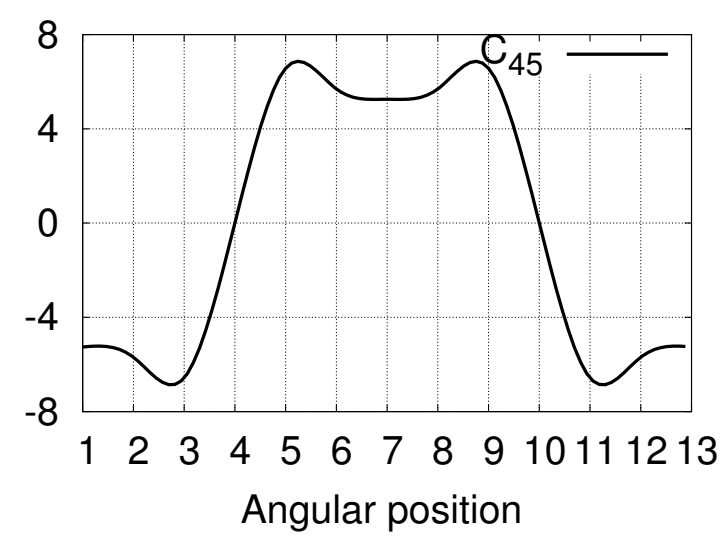

(e)

Figure 3: Variation of coefficients of the stiffness matrix (in GPa) from the $1^{\text {st }}$ step of homogenization with respect to the angle $\theta$ in inner layer. 


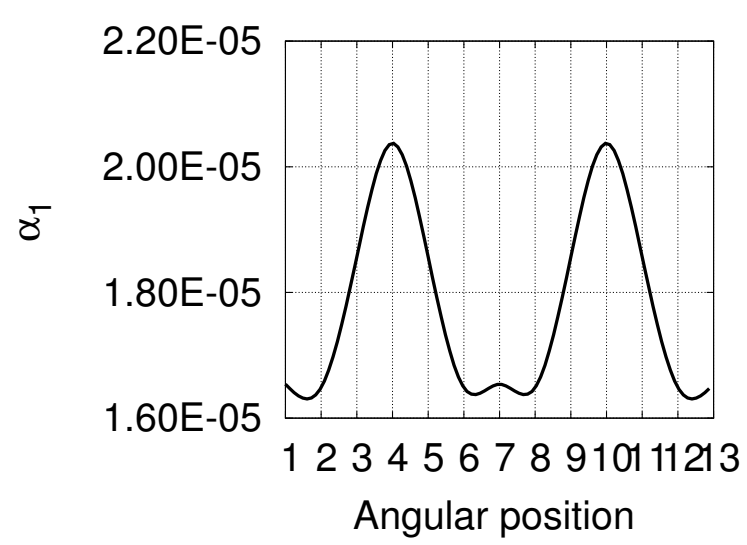

(a)

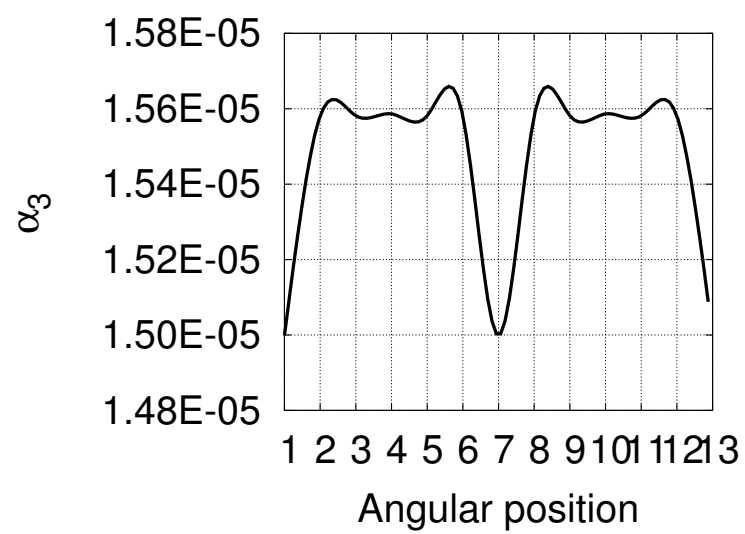

(c)

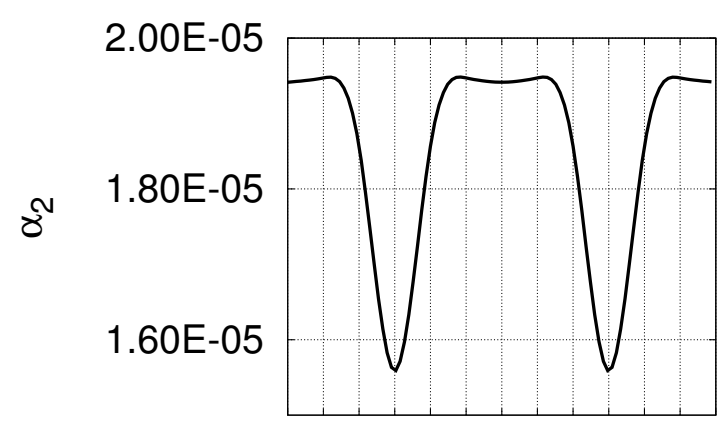

12345678910111213 Angular position

(b)

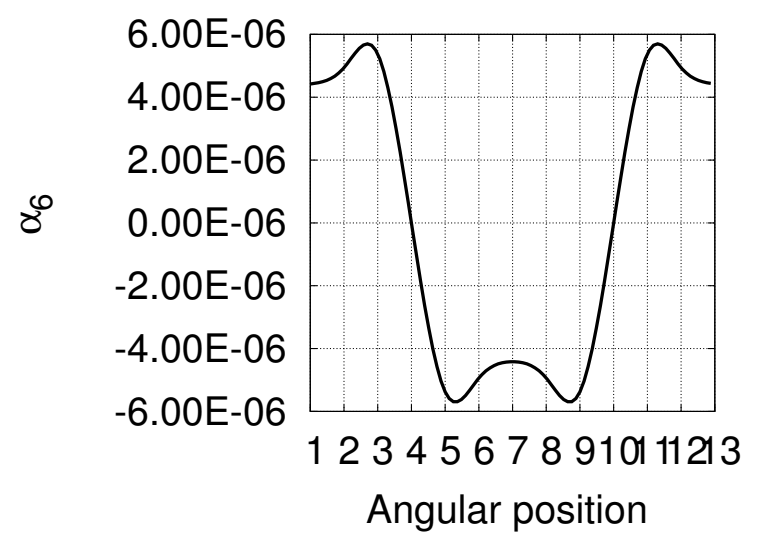

(d)

Figure 4: Variation of coefficients of the thermal expansion tensor (in $\frac{1}{K}$ ) from the $1^{\text {st }}$ step of homogenization in inner layer: (a) $\alpha_{1}$, (b) $\alpha_{2}$, (c) $\alpha_{3}$ and (d) $\alpha_{6}$. In addition $\alpha_{4}=\alpha_{5}=0$. 


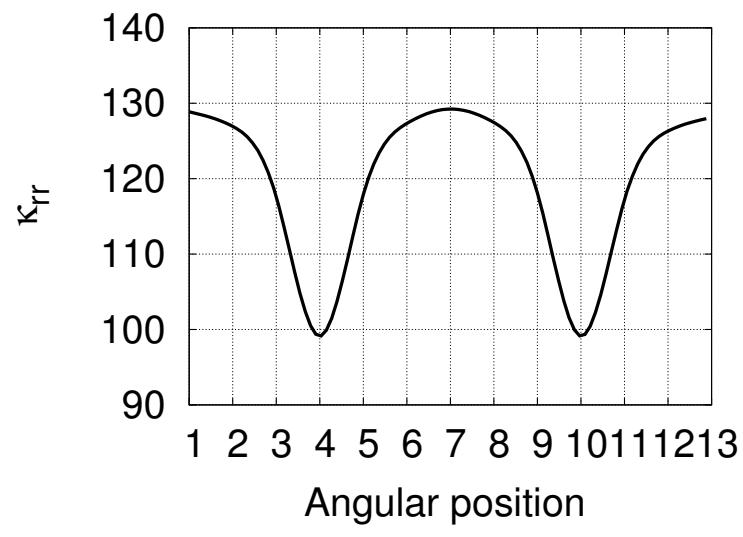

(a)

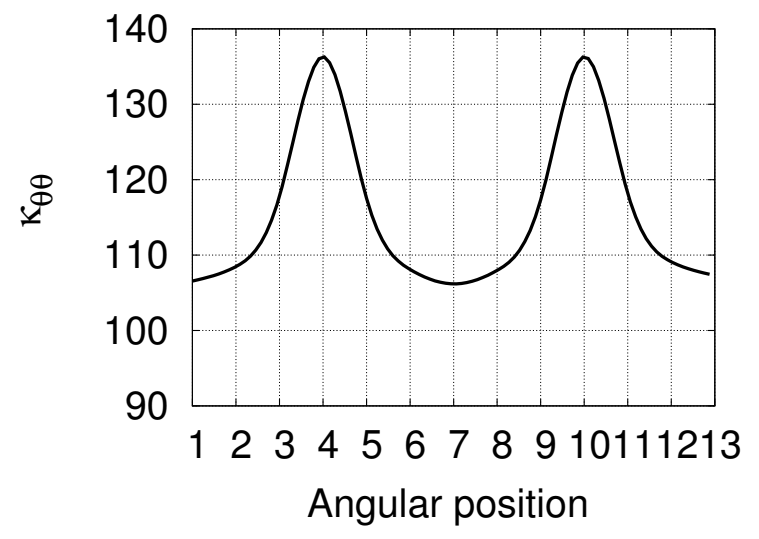

(b)

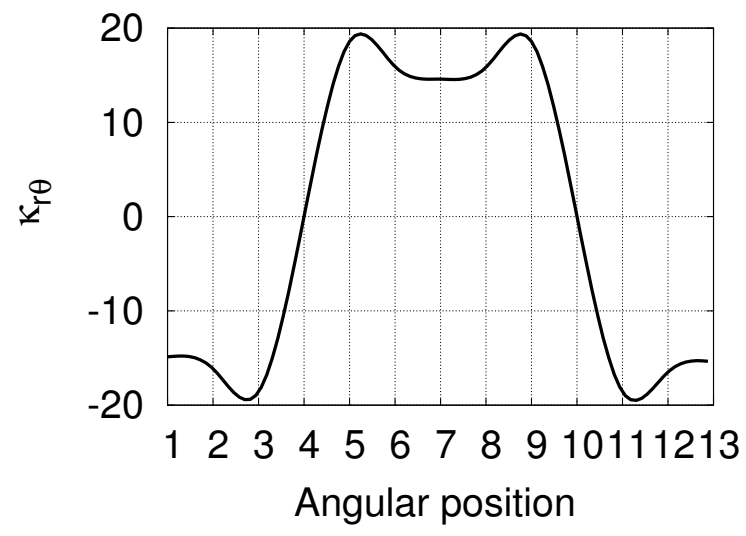

(c)

Figure 5: Variation of coefficients of the thermal conductivity tensor (in $\frac{W}{m K}$ ) from the $1^{s t}$ step of homogenization in inner layer: (a) $\kappa_{r r}$, (b) $\kappa_{\theta \theta}$ and (c) $\kappa_{r \theta}$. In addition $\kappa_{z z}=136.302$ $\frac{W}{m K}$ and $\kappa_{r z}=\kappa_{\theta z}=0$. 
Table 5: Effective symmetric stiffness matrix (in GPa).

$C^{e f f}=\left(\begin{array}{cccccc}171.906 & 85.154 & 82.807 & 0 & 0 & 0 \\ 85.154 & 168.541 & 81.934 & 0 & 0 & 0 \\ 82.807 & 81.934 & 192.606 & 0 & 0 & 0 \\ 0 & 0 & 0 & 45.362 & 0 & 0 \\ 0 & 0 & 0 & 0 & 46.776 & 0 \\ 0 & 0 & 0 & 0 & 0 & 46.207\end{array}\right)$

Table 6: Effective thermal expansion tensor (in $\frac{1}{K}$ ) in inner layer.

$$
\alpha^{e f f}=\left(\begin{array}{c}
17.737 E-6 \\
18.582 E-6 \\
15.448 E-6 \\
0 \\
0 \\
0
\end{array}\right)
$$

In fig. 6 the response of effective radial, hoop and shear (in the cross section) stress vs corresponding strains for $\Delta \mathrm{T}=10 \mathrm{~K}$ is depicted. 


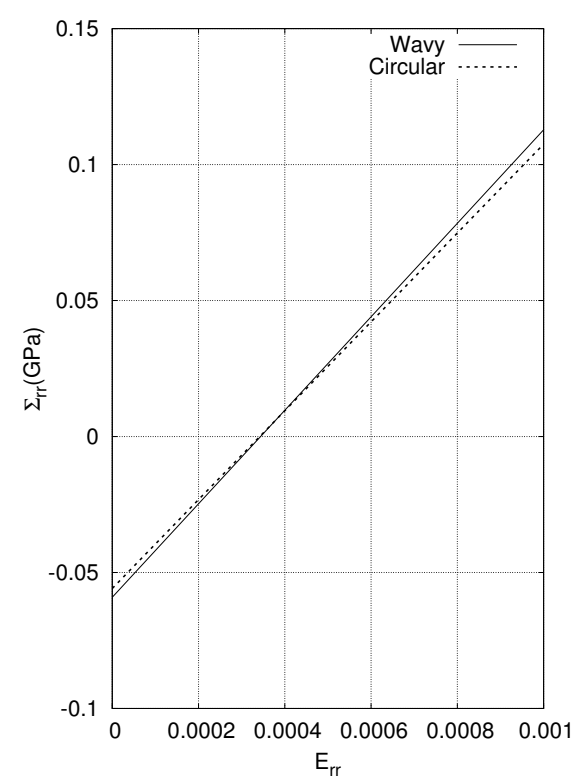

(a)

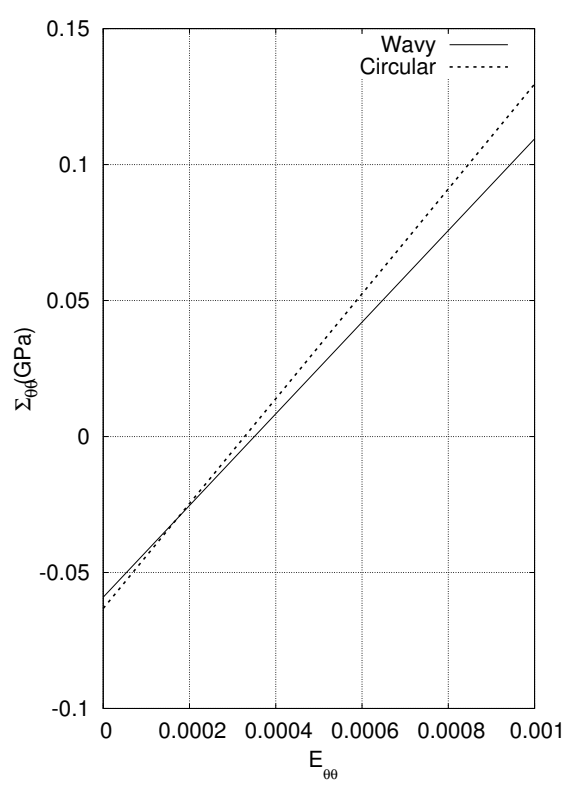

(b)

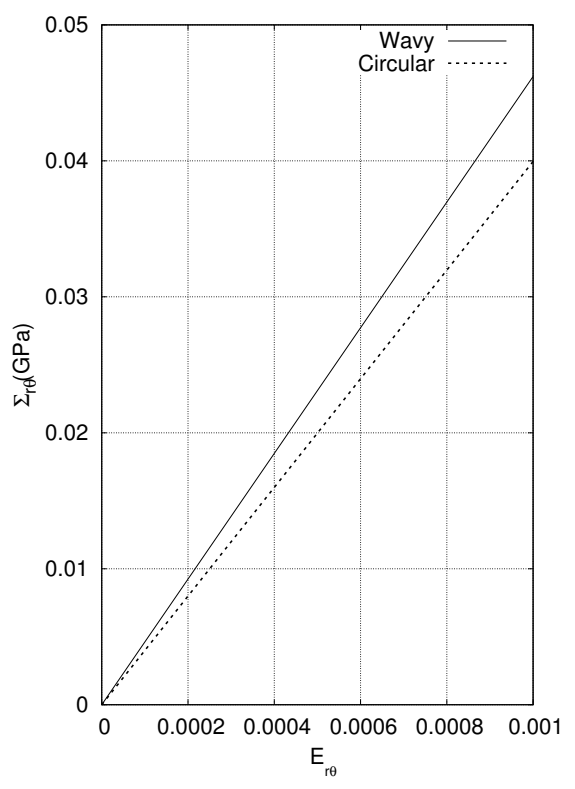

(c)

Figure 6: (a) Effective radial stress vs effective radial strain, (b) effective hoop stress vs effective angular strain and (c) effective shear (in the cross section) stress vs effective shear strain, for $\Delta \mathrm{T}=10 \mathrm{~K}$ in inner layer. 
Table 7: Effective thermal conductivity tensor (in $\frac{W}{m K}$ ) in inner layer.

$$
\kappa^{e f f}=\left(\begin{array}{ccc}
118.146 & 0 & 0 \\
0 & 105.560 & 0 \\
0 & 0 & 136.302
\end{array}\right)
$$




\section{Concluding remarks}

We presented a homogenization technique for a star-shaped cylinder made of numerous thin wavy layers of elastic materials with discontinuous thermomechanical parameters. The waviness of the layers has two major consequences: First, the homogenization needs a two-dimensional cell problem instead of the one-dimensional applied to the wavyless hollow cylinder. Second, the waviness affects the effective thermomechanical behavior in an anisotropic way.

In this paper, we overcome the difficulty of a $2-\mathrm{D}$ cell problem by considering that the structure exhibits, additionally to the periodicity inside every alternating layer, two distinct scales of periodicity, a fine scale in the radial direction and a coarse scale in the angular direction. Then, a multi-scale homogenization method is proposed, starting from the finest scale, that leads to the homogenized coefficients to be used in the next step as heterogenous coefficients.

Both homogenization steps following radial and angular directions use one-dimensional cell problems and give analytical expressions for the coefficients, in terms of the gradient of the wavy layers.

The principal goal of this paper is to apply the above method in order to evaluate the waviness effect to the effective thermomechanical behavior of the tube for any wavy form of the layers. The related results show that waviness affects considerably this behavior, by strengthening the shear resistance, reducing the radial expansion and enhancing the radial conductivity, while weakening the hoop stress capacity, enhancing the angular expansion and reducing the angular conductivity. 
These results could provide guidance for a way to estimate certain difficultto-measure moduli. Moreover, the $1^{\text {st }}$ homogenization step is valid per se as a method for determining the monoclinic behavior of a multilayered wavywalled cylinder. 


\section{Appendix A. Analytical form of cell problems (a) for the $1^{\text {st }}$ step} of homogenization (2.15), (2.16) and (2.19) and (b) for the $2^{\text {nd }}$ step of homogenization (2.33), (2.34) and (2.37)

From the $1^{\text {st }}$ step of homogenization

$$
\begin{aligned}
& \bar{L}_{r}^{(1)} C_{11}+\bar{L}_{r}^{(1)}\left(C_{11} \bar{L}_{r}^{(1)} N_{r}^{1(1)}+C_{12} \bar{L}_{\theta}^{(1)} N_{\theta}^{1(1)}\right)+\bar{L}_{\theta}^{(1)}\left(C_{66} \bar{L}_{r}^{(1)} N_{\theta}^{1(1)}+C_{66} \bar{L}_{\theta}^{(1)} N_{r}^{1}{ }^{(1)}\right)=0 \\
& \bar{L}_{r}^{(1)} C_{12}+\bar{L}_{r}^{(1)}\left(C_{11} \bar{L}_{r}^{(1)} N_{r}^{2(1)}+C_{12} \bar{L}_{\theta}^{(1)} N_{\theta}^{2(1)}\right)+\bar{L}_{\theta}^{(1)}\left(C_{66} \bar{L}_{r}^{(1)} N_{\theta}^{2(1)}+C_{66} \bar{L}_{\theta}^{(1)} N_{r}^{2(1)}\right)=0 \\
& \bar{L}_{r}^{(1)} C_{13}+\bar{L}_{r}^{(1)}\left(C_{11} \bar{L}_{r}^{(1)} N_{r}^{3(1)}+C_{12} \bar{L}_{\theta}^{(1)} N_{\theta}^{3(1)}\right)+\bar{L}_{\theta}^{(1)}\left(C_{66} \bar{L}_{r}^{(1)} N_{\theta}^{3(1)}+C_{66} \bar{L}_{\theta}^{(1)} N_{r}^{3(1)}\right)=0 \\
& \bar{L}_{\theta}^{(1)} C_{21}+\bar{L}_{r}^{(1)}\left(C_{11} \bar{L}_{r}^{(1)} N_{r}^{6(1)}+C_{12} \bar{L}_{\theta}^{(1)} N_{\theta}^{6(1)}\right)+\bar{L}_{\theta}^{(1)}\left(C_{66} \bar{L}_{r}^{(1)} N_{\theta}^{6(1)}+C_{66} \bar{L}_{\theta}^{(1)} N_{r}^{6}{ }^{(1)}\right)=0 \\
& \bar{L}_{r}^{(1)}\left(C_{11} \bar{L}_{r}^{(1)} N_{r}^{5(1)}+C_{12} \bar{L}_{\theta}^{(1)} N_{\theta}^{5(1)}\right)+\bar{L}_{\theta}^{(1)}\left(C_{66} \bar{L}_{r}^{(1)} N_{\theta}^{5(1)}+C_{66} \bar{L}_{\theta}^{(1)} N_{r}^{5(1)}\right)=0 \\
& \bar{L}_{r}^{(1)}\left(C_{11} \bar{L}_{r}^{(1)} N_{r}^{4(1)}+C_{12} \bar{L}_{\theta}^{(1)} N_{\theta}^{4(1)}\right)+\bar{L}_{\theta}^{(1)}\left(C_{66} \bar{L}_{r}^{(1)} N_{\theta}^{4(1)}+C_{66} \bar{L}_{\theta}^{(1)} N_{r}^{4(1)}\right)=0 \\
& \bar{L}_{\theta}^{(1)} C_{21}+\bar{L}_{r}^{(1)}\left(C_{66} \bar{L}_{r}^{(1)} N_{\theta}^{1(1)}+C_{66} \bar{L}_{\theta}^{(1)} N_{r}^{1(1)}\right)+\bar{L}_{\theta}^{(1)}\left(C_{21} \bar{L}_{r}^{(1)} N_{r}^{1(1)}+C_{22} \bar{L}_{\theta}^{(1)} N_{\theta}^{1(1)}\right)=0 \\
& \bar{L}_{\theta}^{(1)} C_{22}+\bar{L}_{r}^{(1)}\left(C_{66} \bar{L}_{r}^{(1)} N_{\theta}^{2(1)}+C_{66} \bar{L}_{\theta}^{(1)} N_{r}^{2(1)}\right)+\bar{L}_{\theta}^{(1)}\left(C_{21} \bar{L}_{r}^{(1)} N_{r}^{2(1)}+C_{22} \bar{L}_{\theta}^{(1)} N_{\theta}^{2(1)}\right)=0 \\
& \bar{L}_{\theta}^{(1)} C_{23}+\bar{L}_{r}^{(1)}\left(C_{66} \bar{L}_{r}^{(1)} N_{\theta}^{3(1)}+C_{66} \bar{L}_{\theta}^{(1)} N_{r}^{3(1)}\right)+\bar{L}_{\theta}^{(1)}\left(C_{21} \bar{L}_{r}^{(1)} N_{r}^{3(1)}+C_{22} \bar{L}_{\theta}^{(1)} N_{\theta}^{3}{ }^{(1)}\right)=0 \\
& \bar{L}_{r}^{(1)} C_{66}+\bar{L}_{r}^{(1)}\left(C_{66} \bar{L}_{r}^{(1)} N_{\theta}^{6(1)}+C_{66} \bar{L}_{\theta}^{(1)} N_{r}^{6(1)}\right)+\bar{L}_{\theta}^{(1)}\left(C_{21} \bar{L}_{r}^{(1)} N_{r}^{6(1)}+C_{22} \bar{L}_{\theta}^{(1)} N_{\theta}^{6}{ }^{(1)}\right)=0 \\
& \bar{L}_{r}^{(1)}\left(C_{66} \bar{L}_{r}^{(1)} N_{\theta}^{5(1)}+C_{66} \bar{L}_{\theta}^{(1)} N_{r}^{5(1)}\right)+\bar{L}_{\theta}^{(1)}\left(C_{21} \bar{L}_{r}^{(1)} N_{r}^{5(1)}+C_{22} \bar{L}_{\theta}^{(1)} N_{\theta}^{5(1)}\right)=0 \\
& \bar{L}_{r}^{(1)}\left(C_{66} \bar{L}_{r}^{(1)} N_{\theta}^{4(1)}+C_{66} \bar{L}_{\theta}^{(1)} N_{r}^{4(r)}\right)+\bar{L}_{\theta}^{(1)}\left(C_{21} \bar{L}_{r}^{(1)} N_{r}^{4(1)}+C_{22} \bar{L}_{\theta}^{(1)} N_{\theta}^{4(1)}\right)=0 \\
& \bar{L}_{r}^{(1)}\left(C_{55} \bar{L}_{r}^{(1)} N_{z}^{4(1)}\right)+\bar{L}_{\theta}^{(1)}\left(C_{44} \bar{L}_{\theta}^{(1)} N_{z}^{4}{ }^{(1)}\right)=0 \\
& \bar{L}_{r}^{(1)}\left(C_{55} \bar{L}_{r}^{(1)} N_{z}^{2(1)}\right)+\bar{L}_{\theta}^{(1)}\left(C_{44} \bar{L}_{\theta}^{(1)} N_{z}^{2(1)}\right)=0 \\
& \bar{L}_{r}^{(1)}\left(C_{55} \bar{L}_{r}^{(1)} N_{z}^{4(1)}\right)+\bar{L}_{\theta}^{(1)}\left(C_{44} \bar{L}_{\theta}^{(1)} N_{z}^{4}{ }^{(1)}\right)=0 \\
& \bar{L}_{r}^{(1)}\left(C_{55} \bar{L}_{r}^{(1)} N_{z}^{6(1)}\right)+\bar{L}_{\theta}^{(1)}\left(C_{44} \bar{L}_{\theta}^{(1)} N_{z}^{6(1)}\right)=0 \\
& \bar{L}_{r}^{(1)} C_{55}+\bar{L}_{r}^{(1)}\left(C_{55} \bar{L}_{r}^{(1)} N_{z}^{5(1)}\right)+\bar{L}_{\theta}^{(1)}\left(C_{44} \bar{L}_{\theta}^{(1)} N_{z}^{5}(1)\right)=0 \\
& \bar{L}_{\theta}^{(1)} C_{44}+\bar{L}_{r}^{(1)}\left(C_{55} \bar{L}_{r}^{(1)} N_{z}^{4(1)}\right)+\bar{L}_{\theta}^{(1)}\left(C_{44} \bar{L}_{\theta}^{(1)} N_{z}^{4}(1)\right)=0
\end{aligned}
$$




$$
\begin{gathered}
\bar{L}_{r}^{(1)} C_{11} a_{1}+\bar{L}_{r}^{(1)} C_{12} a_{2}+\bar{L}_{r}^{(1)} C_{13} a_{3}+ \\
+\left(\bar{L}_{r}^{(1)} C_{11} \bar{L}_{r}^{(1)}+\bar{L}_{\theta}^{(1)} C_{66} \bar{L}_{\theta}^{(1)}\right) N_{r}^{0(1)}+\left(\bar{L}_{r}^{(1)} C_{12} \bar{L}_{\theta}^{(1)}+\bar{L}_{\theta}^{(1)} C_{66} \bar{L}_{r}^{(1)}\right) N_{\theta}^{0}{ }^{(1)}=0, \\
\bar{L}_{\theta}^{(1)} C_{21} a_{1}+\bar{L}_{\theta}^{(1)} C_{22} a_{2}+\bar{L}_{\theta}^{(1)} C_{23} a_{3}+ \\
+\left(\bar{L}_{r}^{(1)} C_{66} \bar{L}_{\theta}^{(1)}+\bar{L}_{\theta}^{(1)} C_{21} \bar{L}_{r}^{(1)}\right) N_{r}^{0(1)}+\left(\bar{L}_{r}^{(1)} C_{66} \bar{L}_{r}^{(1)}+\bar{L}_{\theta}^{(1)} C_{22} \bar{L}_{\theta}^{(1)}\right) N_{\theta}^{0(1)}=0, \\
\left(\bar{L}_{r}^{(1)} C_{55} \bar{L}_{r}^{(1)}+\bar{L}_{\theta}^{(1)} C_{44} \bar{L}_{\theta}^{(1)}\right) N_{z}^{0(1)}=0,
\end{gathered}
$$

or

$$
\begin{gathered}
\frac{\partial}{\partial \varrho}\left(A_{3}^{(1)}+A_{1}^{(1)} \frac{\partial N_{r}^{0(1)}}{\partial \bar{\varrho}}+A_{2}^{(1)} \frac{\partial N_{\theta}^{0(1)}}{\partial \bar{\varrho}}\right)=0 \\
\frac{\partial}{\partial \bar{\varrho}}\left(B_{3}^{(1)}+B_{1}^{(1)} \frac{\partial N_{r}^{0(1)}}{\partial \bar{\varrho}}+B_{2}^{(1)} \frac{\partial N_{\theta}^{0(1)}}{\partial \bar{\varrho}}\right)=0 \\
\frac{\partial}{\partial \bar{\varrho}}\left(D_{1}^{(1)} \frac{\partial N_{z}^{0(1)}}{\partial \bar{\varrho}}\right)=0
\end{gathered}
$$

where

$$
\begin{gathered}
A_{1}^{(1)}=P_{1}^{(1)} C_{11} P_{1}^{(1)}+P_{2}^{(1)} C_{66} P_{2}^{(1)}, \\
A_{2}^{(1)}=P_{1}^{(1)} C_{12} P_{2}^{(1)}+P_{2}^{(1)} C_{66} P_{1}^{(1)}, \\
A_{3}^{(1)}=P_{1}^{(1)} C_{11} a_{1}+P_{1}^{(1)} C_{12} a_{2}+P_{1}^{(1)} C_{13} a_{3}, \\
B_{1}^{(1)}=P_{1}^{(1)} C_{66} P_{2}^{(1)}+P_{1}^{(1)} C_{21} P_{1}^{(1)}, \\
B_{2}^{(1)}=P_{1}^{(1)} C_{66} P_{1}^{(1)}+P_{2}^{(1)} C_{22} P_{2}^{(1)}, \\
B_{3}^{(1)}=P_{2}^{(1)} C_{21} a_{1}+P_{2}^{(1)} C_{22} a_{2}+P_{2}^{(1)} C_{23} a_{3}, \\
D_{1}^{(1)}=P_{1}^{(1)} C_{55} P_{1}^{(1)}+P_{2}^{(1)} C_{44} P_{2}^{(1)},
\end{gathered}
$$


and

$$
\begin{aligned}
& \bar{L}_{r}^{(1)} \kappa_{r r}+\bar{L}_{\theta}^{(1)} \kappa_{\theta r}+\left(\bar{L}_{r}^{(1)} \kappa_{r r} \bar{L}_{r}^{(1)}+\bar{L}_{r}^{(1)} \kappa_{r \theta} \bar{L}_{\theta}^{(1)}+\bar{L}_{\theta}^{(1)} \kappa_{\theta r} \bar{L}_{r}^{(1)}+\bar{L}_{\theta}^{(1)} \kappa_{\theta \theta} \bar{L}_{\theta}^{(1)}\right) \frac{\partial W_{r}^{(1)}}{\partial \varrho}=0, \\
& \bar{L}_{r}^{(1)} \kappa_{r \theta}+\bar{L}_{\theta}^{(1)} \kappa_{\theta \theta}+\left(\bar{L}_{r}^{(1)} \kappa_{r r} \bar{L}_{r}^{(1)}+\bar{L}_{r}^{(1)} \kappa_{r \theta} \bar{L}_{\theta}^{(1)}+\bar{L}_{\theta}^{(1)} \kappa_{\theta r} \bar{L}_{r}^{(1)}+\bar{L}_{\theta}^{(1)} \kappa_{\theta \theta} \bar{L}_{\theta}^{(1)}\right) \frac{\partial W_{\theta}^{(1)}}{\partial \bar{\varrho}}=0, \\
& \bar{L}_{r}^{(1)} \kappa_{r z}+\bar{L}_{\theta}^{(1)} \kappa_{\theta z}+\left(\bar{L}_{r}^{(1)} \kappa_{r r} \bar{L}_{r}^{(1)}+\bar{L}_{r}^{(1)} \kappa_{r \theta} \bar{L}_{\theta}^{(1)}+\bar{L}_{\theta}^{(1)} \kappa_{\theta r} \bar{L}_{r}^{(1)}+\bar{L}_{\theta}^{(1)} \kappa_{\theta \theta} \bar{L}_{\theta}^{(1)}\right) \frac{\partial W_{z}^{(1)}}{\partial \varrho}=0,
\end{aligned}
$$

or

$$
\begin{aligned}
& \frac{\partial}{\partial \bar{\varrho}}\left(F_{1}^{(1)}+F_{0}^{(1)} \frac{\partial W_{r}^{(1)}}{\partial \bar{\varrho}}\right)=0, \\
& \frac{\partial}{\partial \bar{\varrho}}\left(F_{2}^{(1)}+F_{0}^{(1)} \frac{\partial W_{\theta}^{(1)}}{\partial \bar{\varrho}}\right)=0 \\
& \frac{\partial}{\partial \bar{\varrho}}\left(F_{3}^{(1)}+F_{0}^{(1)} \frac{\partial W_{z}^{(1)}}{\partial \bar{\varrho}}\right)=0
\end{aligned}
$$

where

$$
\begin{gathered}
F_{0}^{(1)}=P_{1}^{(1)} \kappa_{r r} P_{1}^{(1)}+P_{1}^{(1)} \kappa_{r \theta} P_{2}^{(1)}+P_{2}^{(1)} \kappa_{\theta r} P_{1}^{(1)}+P_{2}^{(1)} \kappa_{\theta \theta} P_{2}^{(1)}, \\
F_{1}^{(1)}=P_{1}^{(1)} \kappa_{r r}+P_{2}^{(1)} \kappa_{\theta r}, \\
F_{2}^{(1)}=P_{1}^{(1)} \kappa_{r \theta}+P_{2}^{(1)} \kappa_{\theta \theta}, \\
F_{3}^{(1)}=P_{1}^{(1)} \kappa_{r z}+P_{2}^{(1)} \kappa_{\theta z} .
\end{gathered}
$$


From the $2^{\text {nd }}$ step of homogenization

$$
\begin{aligned}
& \bar{L}_{\theta}^{(2)} C_{61}^{e f f(1)}+\bar{L}_{\theta}^{(2)} C_{66}^{e f f(1)} \bar{L}_{\theta}^{(2)} N_{r}^{1{ }^{(2)}}+\bar{L}_{\theta}^{(2)} C_{62}^{e f f(1)} \bar{L}_{\theta}^{(2)} N_{\theta}^{1(2)}=0 \\
& \bar{L}_{\theta}^{(2)} C_{62}^{e f f(1)}+\bar{L}_{\theta}^{(2)} C_{66}^{e f f(1)} \bar{L}_{\theta}^{(2)} N_{r}{ }^{(2)}+\bar{L}_{\theta}^{(2)} C_{62}^{e f f(1)} \bar{L}_{\theta}^{(2)} N_{\theta}{ }^{(2)}=0 \\
& \bar{L}_{\theta}^{(2)} C_{63}^{e f f(1)}+\bar{L}_{\theta}^{(2)} C_{66}^{e f f(1)} \bar{L}_{\theta}^{(2)} N_{r}^{3{ }^{(2)}}+\bar{L}_{\theta}^{(2)} C_{62}^{e f f(1)} \bar{L}_{\theta}^{(2)} N_{\theta}^{3(2)}=0
\end{aligned}
$$

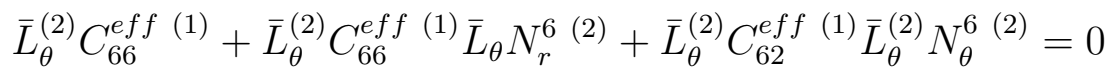

$$
\begin{aligned}
& \bar{L}_{\theta}^{(2)} C_{66}^{e f f(1)} \bar{L}_{\theta}^{(2)} N_{r}^{4(2)}+\bar{L}_{\theta}^{(2)} C_{62}^{e f f(1)} \bar{L}_{\theta}^{(2)} N_{\theta}^{4(2)}=0 \\
& \bar{L}_{\theta}^{(2)} C_{66}^{e f f(1)} \bar{L}_{\theta}^{(2)} N_{r}^{5}{ }^{(2)}+\bar{L}_{\theta}^{(2)} C_{62}^{e f f(1)} \bar{L}_{\theta}^{(2)} N_{\theta}^{5(2)}=0 \\
& \bar{L}_{\theta}^{(2)} C_{21}^{e f f(1)}+\bar{L}_{\theta}^{(2)} C_{22}^{e f f(1)} \bar{L}_{\theta} N_{\theta}^{1{ }^{(2)}}+\bar{L}_{\theta}^{(2)} C_{26}^{e f f(1)} \bar{L}_{\theta}^{(2)} N_{r}^{1(2)}=0 \\
& \bar{L}_{\theta}^{(2)} C_{22}^{e f f(1)}+\bar{L}_{\theta}^{(2)} C_{22}^{e f f(1)} \bar{L}_{2} N_{\theta}^{2(2)}+\bar{L}_{\theta}^{(2)} C_{26}^{e f f(1)} \bar{L}_{\theta}^{(2)} N_{r}^{2(2)}=0 \\
& \bar{L}_{\theta}^{(2)} C_{23}^{e f f(1)}+\bar{L}_{\theta}^{(2)} C_{22}^{e f f(1)} \bar{L}_{\theta} N_{\theta}^{3(2)}+\bar{L}_{\theta}^{(2)} C_{26}^{e f f(1)} \bar{L}_{\theta}^{(2)} N_{r}^{3}{ }^{(2)}=0 \\
& \bar{L}_{\theta}^{(2)} C_{26}^{e f f(1)}+\bar{L}_{\theta}^{(2)} C_{22}^{e f f(1)} \bar{L}_{\theta}^{(2)} N_{\theta}^{6(2)}+\bar{L}_{\theta}^{(2)} C_{26}^{e f f(1)} \bar{L}_{2}^{(\theta)} N_{r}^{6(2)}=0 \\
& \bar{L}_{\theta}^{(2)} C_{22}^{e f f(1)} \bar{L}_{\theta}^{(2)} N_{\theta}^{4(2)}+\bar{L}_{\theta}^{(2)} C_{26}^{e f f(1)} \bar{L}_{\theta}^{(2)} N_{r}^{4}{ }^{(2)}=0 \\
& \bar{L}_{\theta}^{(2)} C_{22}^{e f f(1)} \bar{L}_{\theta}^{(2)} N_{\theta}^{5}{ }^{(2)}+\bar{L}_{\theta}^{(2)} C_{26}^{e f f(1)} \bar{L}_{\theta}^{(2)} N_{r}^{5}{ }^{(2)}=0 \\
& \bar{L}_{\theta}^{(2)} C_{44}^{e f f(1)} \bar{L}_{\theta}^{(2)} N_{z}^{1(2)}=0 \\
& \bar{L}_{\theta}^{(2)} C_{44}^{e f f(1)} \bar{L}_{\theta}^{(2)} N_{z}^{2(2)}=0 \\
& \bar{L}_{\theta}^{(2)} C_{44}^{e f f(1)} \bar{L}_{\theta}^{(2)} N_{z}^{3(2)}=0 \\
& \bar{L}_{\theta}^{(2)} C_{44}^{e f f(1)} \bar{L}_{\theta}^{(2)} N_{z}^{6(2)}=0 \\
& \bar{L}_{\theta}^{(2)} C_{44}^{e f f(1)}+\bar{L}_{\theta}^{(2)} C_{44}^{e f f(1)} \bar{L}_{\theta} N_{z}^{4(2)}=0 \\
& \bar{L}_{\theta}^{(2)} C_{45}^{e f f(1)}+\bar{L}_{\theta}^{(2)} C_{44}^{e f f(1)} \bar{L}_{\theta}^{(2)} N_{z}^{5(2)}=0
\end{aligned}
$$




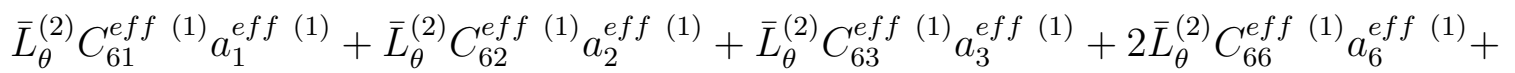

$$
\begin{aligned}
& +\bar{L}_{\theta}^{(2)} C_{66}^{e f f(1)} \bar{L}_{\theta}^{(2)} N_{r}^{0(2)}+\bar{L}_{\theta}^{(2)} C_{62}^{e f f(1)} \bar{L}_{\theta}^{(2)} N_{\theta}^{0(2)}=0
\end{aligned}
$$

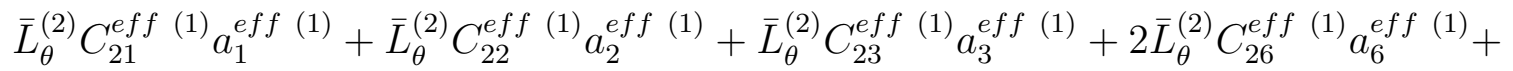

$$
\begin{aligned}
& +\bar{L}_{\theta}^{(2)} C_{26}^{e f f(1)} \bar{L}_{\theta}^{(2)} N_{r}^{0(2)}+\bar{L}_{2}^{(2)} C_{22}^{e f f(1)} \bar{L}_{\theta}^{(2)} N_{\theta}^{0(2)}=0, \\
& 2 \bar{L}_{\theta}^{(2)} C_{45}^{e f f(1)} a_{5}^{e f f(1)}+2 \bar{L}_{\theta}^{(2)} C_{44}^{e f f(1)} a_{4}^{e f f(1)}+\bar{L}_{\theta}^{(2)} C_{44}^{e f f(1)} \bar{L}_{\theta}^{(2)} N_{z}^{0(2)}=0,
\end{aligned}
$$

or

$$
\begin{gathered}
\frac{\partial}{\partial \bar{\theta}}\left(A_{3}^{(2)}+A_{1}^{(2)} \frac{\partial N_{r}^{0(2)}}{\partial \bar{\varrho}}+A_{2}^{(2)} \frac{\partial N_{\theta}^{0(2)}}{\partial \bar{\varrho}}\right)=0 \\
\frac{\partial}{\partial \bar{\theta}}\left(B_{3}^{(2)}+B_{1}^{(2)} \frac{\partial N_{r}^{0(2)}}{\partial \bar{\varrho}}+B_{2}^{(2)} \frac{\partial N_{\theta}^{0(2)}}{\partial \bar{\varrho}}\right)=0 \\
\frac{\partial}{\partial \bar{\theta}}\left(D_{2}^{(2)}+D_{1}^{(2)} \frac{\partial N_{z}^{0(2)}}{\partial \varrho}\right)=0
\end{gathered}
$$

where

$$
\begin{aligned}
& A_{1}^{(2)}=P_{2}^{(2)} C_{66}^{e f f(1)} P_{2}^{(2)}, \\
& A_{2}^{(2)}=P_{2}^{(2)} C_{62}^{e f f(1)} P_{2}^{(2)}, \\
& A_{3}^{(2)}=P_{2}^{(2)} C_{61}^{e f f(1)} a_{1}^{e f f(1)}+P_{2}^{(2)} C_{62} a_{2}^{e f f(1)}+P_{2}^{(2)} C_{63}^{e f f(1)} a_{3}^{e f f(1)}+2 P_{2}^{(2)} C_{66}^{e f f(1)} a_{6} \text {, } \\
& B_{1}^{(2)}=P_{2}^{(2)} C_{26}^{e f f(1)} P_{2}^{(2)}, \\
& B_{2}^{(2)}=P_{2}^{(2)} C_{22}^{e f f(1)} P_{2}^{(2)} \\
& B_{3}^{(2)}=P_{2}^{(2)} C_{21}^{e f f(1)} a_{1}^{e f f(1)}+P_{2}^{(2)} C_{22} a_{2}^{e f f(1)}+P_{2}^{(2)} C_{23} a_{3}^{e f f(1)}+2 P_{2}^{(2)} C_{26}^{e f f(1)} a_{6}^{e f f(1)}, \\
& D_{1}^{(2)}=P_{2}^{(2)} C_{44}^{e f f(1)} P_{2}^{(2)}, \\
& D_{2}^{(2)}=2 P_{2}^{(2)} C_{45}^{e f f(1)} a_{5}^{e f f(1)}+2 P_{2}^{(2)} C_{44} a_{4}^{e f f(1)} \text {. }
\end{aligned}
$$




$$
\begin{aligned}
& \bar{L}_{\theta}^{(2)} \kappa_{\theta r}^{e f f(1)}+\bar{L}_{\theta}^{(2)} \kappa_{\theta \theta}^{e f f(1)} \bar{L}_{\theta}^{(2)} \frac{\partial W_{r}^{(2)}}{\partial \bar{\varrho}}=0 \\
& \bar{L}_{\theta}^{(2)} \kappa_{\theta \theta}^{e f f(1)}+\bar{L}_{\theta}^{(2)} \kappa_{\theta \theta}^{e f f(1)} \bar{L}_{\theta}^{(2)} \frac{\partial W_{\theta}^{(2)}}{\partial \bar{\varrho}}=0 \\
& \bar{L}_{\theta}^{(2)} \kappa_{\theta z}^{e f f(1)}+\bar{L}_{\theta}^{(2)} \kappa_{\theta \theta}^{e f f(1)} \bar{L}_{\theta}^{(2)} \frac{\partial W_{z}^{(2)}}{\partial \bar{\varrho}}=0
\end{aligned}
$$

or

$$
\begin{aligned}
& \frac{\partial}{\partial \bar{\varrho}}\left(F_{1}^{(2)}+F_{0}^{(2)} \frac{\partial W_{r}^{(2)}}{\partial \bar{\varrho}}\right)=0, \\
& \frac{\partial}{\partial \bar{\varrho}}\left(F_{2}^{(2)}+F_{0}^{(2)} \frac{\partial W_{\theta}^{(2)}}{\partial \bar{\varrho}}\right)=0 \\
& \frac{\partial}{\partial \bar{\varrho}}\left(F_{3}^{(2)}+F_{0}^{(2)} \frac{\partial W_{z}^{(2)}}{\partial \bar{\varrho}}\right)=0
\end{aligned}
$$

where

$$
\begin{gathered}
F_{0}^{(2)}=P_{2}^{(2)} \kappa_{\theta \theta}^{\text {eff }{ }^{(1)}} P_{2}^{(2)}, \\
F_{1}^{(2)}=P_{2}^{(2)} \kappa_{\theta r}^{\text {eff }(1)}, \\
F_{2}^{(2)}=P_{2}^{(2)} \kappa_{\theta \theta}^{\text {eff }(1)} \\
F_{3}^{(2)}=P_{2}^{(2)} \kappa_{\theta z}^{\text {eff }(1)}
\end{gathered}
$$


Appendix B. Solutions of cell problems (a) from the $1^{\text {st }}$ step of homogenization (2.15), (2.16) and (2.19) and (b) from the $2^{\text {nd }}$ step of homogenization (2.33), (2.34) and (2.37)

From the $1^{\text {st }}$ step of homogenization

$$
\begin{aligned}
& \mathrm{A}_{1}^{(i)(1)}=P_{1}^{(1)} C_{66}^{(i)} P_{1}^{(1)}+P_{2}^{(1)} C_{22}^{(i)} P_{2}^{(1)}, \\
& \mathrm{A}_{2}^{(i)(1)}=P_{1}^{(1)} C_{66}^{(i)} P_{2}^{(1)}+P_{2}^{(1)} C_{21}^{(i)} P_{1}^{(1)}, \\
& \mathrm{B}_{1}^{(i)(1)}=P_{1}^{(1)} C_{11}^{(i)} P_{1}^{(1)}+P_{2}^{(1)} C_{66}^{(i)} P_{2}^{(1)}, \\
& \mathrm{B}_{2}^{(i)(1)}=P_{1}^{(1)} C_{12}^{(i)} P_{2}^{(1)}+P_{2}^{(1)} C_{66}^{(i)} P_{1}^{(1)},
\end{aligned}
$$

for $a=1,2,3,4,5,6$, where $n$ are the phases of the structure,

$$
\begin{gathered}
\lambda_{1}^{a(1)}=\frac{\mathrm{Q}_{3}^{a}{ }^{(1)} \mathrm{R}_{2}^{(1)}-\mathrm{R}_{3}^{a(1)} \mathrm{Q}_{2}^{(1)}}{\mathrm{Q}_{1}^{(1)} \mathrm{R}_{2}^{(1)}-\mathrm{R}_{1}^{(1)} \mathrm{Q}_{2}^{(1)}}, \\
\lambda_{2}^{a(1)}=\frac{\mathrm{Q}_{1}^{(1)} \mathrm{R}_{3}^{a(1)}-\mathrm{R}_{1}^{(1)} \mathrm{Q}_{3}^{a}{ }^{(1)}}{\mathrm{Q}_{1}^{(1)} \mathrm{R}_{2}^{(1)}-\mathrm{R}_{1}^{(1)} \mathrm{Q}_{2}^{(1)}}, \\
\lambda_{3}^{a(1)}=\frac{\sum_{i=1}^{n} \frac{\left(P_{1}^{(1)} C_{5 a}^{(i)}+P_{2}^{(1)} C_{4 a}^{(i)} c^{(i)}\right.}{P_{1}^{(1)} C_{55}^{(i)} P_{1}^{(1)}+P_{2}^{(1)} C_{44}^{(i)} P_{2}^{(1)}}}{\sum_{i=1}^{n} \frac{c^{(i)}}{P_{1}^{(1)} C_{55}^{(i)} P_{1}^{(1)}+P_{2}^{(1)} C_{44}^{(i)} P_{2}^{(1)}}}
\end{gathered}
$$


where

$$
\begin{aligned}
& \mathrm{Q}_{1}^{(1)}=\sum_{i=1}^{n} \frac{\mathrm{B}_{2}^{(i)}{ }^{(1)} c^{(i)}}{\mathrm{A}_{1}^{(i)(1)} \mathrm{B}_{2}^{(i)(1)}-\mathrm{A}_{2}^{(i)(1)} \mathrm{B}_{1}^{(i)(1)}} \\
& \mathrm{Q}_{2}^{(1)}=-\sum_{i=1}^{n} \frac{\mathrm{A}_{2}^{(i)(1)} c^{(i)}}{\mathrm{A}_{1}^{(i)(1)} \mathrm{B}_{2}^{(i)(1)}-\mathrm{A}_{2}^{(i)(1)} \mathrm{B}_{1}^{(i)(1)}} \\
& \mathrm{Q}_{3}^{a(1)}=\sum_{i=1}^{n} \frac{\left(P_{1}^{(1)} C_{6 a}^{(i)}+P_{2}^{(1)} C_{2 a}^{(i)}\right) B_{2}^{(i)}{ }^{(1)} c^{(i)}}{\mathrm{A}_{1}^{(i)}{ }^{(1)} \mathrm{B}_{2}^{(i)}{ }^{(1)}-\mathrm{A}_{2}^{(i)}{ }^{(1)} \mathrm{B}_{1}^{(i)}{ }^{(1)}}-\sum_{i=1}^{n} \frac{\left(P_{1}^{(1)} C_{1 a}^{(i)}+P_{2}^{(1)} C_{2 a}^{(i)}\right) \mathrm{A}_{2}^{(i)}{ }^{(1)} c^{(i)}}{\mathrm{A}_{1}^{(i)}{ }^{(1)} \mathrm{B}_{2}^{(i)}{ }^{(1)}-\mathrm{A}_{2}^{(i)}{ }^{(1)} \mathrm{B}_{1}^{(i)}{ }^{(1)}}, \\
& \mathrm{R}_{1}^{(1)}=-\sum_{i=1}^{n} \frac{\mathrm{B}_{1}^{(i)(1)} c^{(i)}}{\mathrm{A}_{1}^{(i)(1)} \mathrm{B}_{2}^{(i)(1)}-\mathrm{A}_{2}^{(i)(1)} \mathrm{B}_{1}^{(i)(1)}} \\
& \mathrm{R}_{2}^{(1)}=\sum_{i=1}^{n} \frac{A_{1}^{(i)(1)} c^{(i)}}{\mathrm{A}_{1}^{(i)(1)} \mathrm{B}_{2}^{(i)(1)}-\mathrm{A}_{2}^{(i)(1)} \mathrm{B}_{1}^{(i)(1)}} \\
& \mathrm{R}_{3}^{a(1)}=\sum_{i=1}^{n} \frac{\left(P_{1}^{(1)} C_{1 a}^{(i)}+P_{2}^{(1)} C_{2 a}^{(i)}\right) B_{1}^{(i)}{ }^{(1)} c^{(i)}}{\mathrm{A}_{1}^{(i)}{ }^{(1)} \mathrm{B}_{2}^{(i)(1)}-\mathrm{A}_{2}^{(i)}{ }^{(1)} \mathrm{B}_{1}^{(i)}(1)}-\sum_{i=1}^{n} \frac{\left(P_{1}^{(1)} C_{6 a}^{(i)}+P_{2}^{(1)} C_{2 a}^{(i)}\right) \mathrm{A}_{1}^{(i)}{ }^{(1)} c^{i}}{\mathrm{~A}_{1}^{(i)}{ }^{(1)} \mathrm{B}_{2}^{(i)}{ }^{(1)}-\mathrm{A}_{2}^{(i)}{ }^{(1)} \mathrm{B}_{1}^{(i)}{ }^{(1)}} \\
& \bar{\lambda}_{1}^{(1)}=\frac{K_{3}^{(1)} M_{2}^{(1)}-K_{2}^{(1)} M_{3}^{(1)}}{K_{1}^{(1)} M_{2}^{(1)}-K_{2}^{(1)} M_{1}^{(1)}} \\
& \bar{\lambda}_{2}^{(1)}=\frac{K_{1}^{(1)} M_{3}^{(1)}-K_{3}^{(1)} M_{1}^{(1)}}{K_{1}^{(1)} M_{2}^{(1)}-K_{2}^{(1)} M_{1}^{(1)}} \\
& \bar{\lambda}_{3}^{(1)}=\frac{\sum_{i=1}^{n} \frac{D_{2}^{(i)(1)} c^{(i)}}{D_{1}^{(i)(1)}}}{\sum_{i=1}^{n} \frac{c^{(i)}}{D_{1}^{(i)(1)}}}
\end{aligned}
$$


where

$$
\begin{aligned}
& K_{1}^{(1)}=\sum_{i=1}^{n} \frac{B_{2}^{(i)(1)} c^{(i)}}{A_{1}^{(i)(1)} B_{2}^{(i)(1)}-A_{2}^{(i)(1)} B_{1}^{(i)(1)}}, \\
& K_{2}^{(1)}=-\sum_{i=1}^{n} \frac{A_{2}^{(i)}{ }^{(1)} c^{(i)}}{A_{1}^{(i)(1)(i)(1)}-A_{2}^{(i)}{ }^{(1)} B_{1}^{(i)(1)}},
\end{aligned}
$$

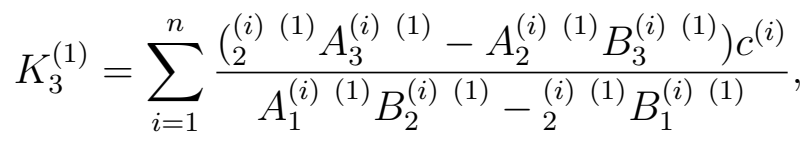

$$
\begin{aligned}
& M_{1}^{(1)}=-\sum_{i=1}^{n} \frac{B_{1}^{(i)}{ }^{(1)} c^{(i)}}{A_{1}^{(i){ }^{(1)}} B_{2}^{(i)(1)}-A_{2}^{(i)(1)} B_{1}^{(i)(1)}}, \\
& M_{2}^{(1)}=\sum_{i=1}^{n} \frac{A_{1}^{(i)(1)} c^{(i)}}{A_{1}^{(i)(1)} B_{2}^{(i)(1)}-A_{2}^{(i)(1)} B_{1}^{(i)(1)}},
\end{aligned}
$$

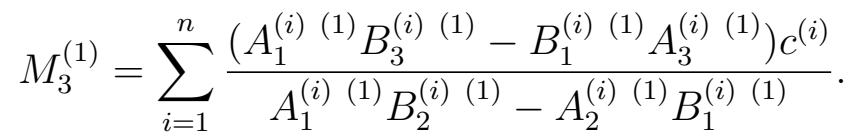

$$
\begin{aligned}
& \tilde{\lambda}_{1}^{(1)}=\frac{\sum_{i=1}^{n} \frac{F_{1}^{(i)(1)} c^{(i)}}{F_{0}^{(i)(1)}}}{\sum_{i=1}^{n} \frac{c^{(i)}}{F_{0}^{(i)(1)}}} \\
& \tilde{\lambda}_{2}^{(1)}=\frac{\sum_{i=1}^{n} \frac{F_{2}^{(i)(1)} c^{(i)}}{F_{0}^{(i)(1)}}}{\sum_{i=1}^{n} \frac{c^{(i)}}{F_{0}^{(i)(1)}}} \\
& \tilde{\lambda}_{3}^{(1)}=\frac{\sum_{i=1}^{n} \frac{F_{3}^{(i)(1)} c^{(i)}}{F_{0}^{(i)(1)}}}{\sum_{i=1}^{n} \frac{c^{(i)}}{F_{0}^{(i)(1)}}}
\end{aligned}
$$


From the $2^{\text {nd }}$ step of homogenization

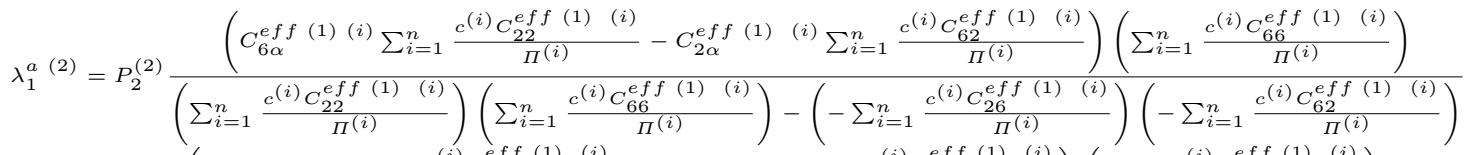

$$
\begin{aligned}
& +P_{2}^{(2)} \frac{-\left(C_{6 \alpha}^{e f f(1)}{ }^{(i)} \sum_{i=1}^{n} \frac{c^{(i)} C_{26}^{e f f}(1)(i)}{\Pi^{(i)}}+C_{2 \alpha}^{e f f(1)}{ }^{(i)} \sum_{i=1}^{n} \frac{c^{(i)} C_{62}^{e f f}(1)(i)}{\Pi^{(i)}}\right)\left(\sum_{i=1}^{n} \frac{c^{(i)} C_{62}^{e f f}(1) \quad(i)}{\Pi^{(i)}}\right)}{\left(\sum_{i=1}^{n} \frac{c^{(i)} C_{22}^{e f f}(1){ }^{(i)}}{\Pi^{(i)}}\right)\left(\sum_{i=1}^{n} \frac{c^{(i)} C_{66}^{e f f}(1)(i)}{\Pi^{(i)}}\right)-\left(-\sum_{i=1}^{n} \frac{c^{(i)} C_{26}^{e f f}(1)(i)}{\Pi^{(i)}}\right)\left(-\sum_{i=1}^{n} \frac{c^{(i)} C_{62}^{e f f}(1)(i)}{\Pi^{(i)}}\right)},
\end{aligned}
$$

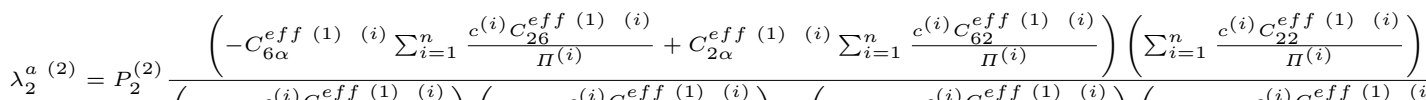

$$
\begin{aligned}
& \left(\sum_{i=1}^{n} \frac{c^{(i)} C_{22}^{e f f(1)}(i)}{\Pi^{(i)}}\right)\left(\sum_{i=1}^{n} \frac{c^{(i)} C_{66}^{e f f(1)}(i)}{\Pi^{(i)}}\right)-\left(-\sum_{i=1}^{n} \frac{c^{(i)} C_{26}^{e f f(1)}(i)}{\Pi^{(i)}}\right)\left(-\sum_{i=1}^{n} \frac{c^{(i)} C_{62}^{e f f(1)} \Pi^{(i)}}{\Pi^{(i)}}\right)
\end{aligned}
$$

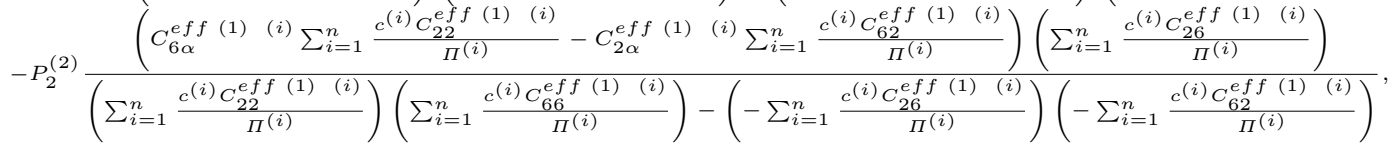

$$
\begin{aligned}
& \lambda_{3}^{a(2)}=P_{2}^{(2)} \frac{\sum_{i=1}^{n} \frac{c^{(i)} C_{4 \alpha}^{e f f(1)(i)}}{C_{44}^{e f f(1)(i)}}}{\sum_{i=1}^{n} \frac{c^{(i)}}{C_{44}^{e f f(1)(i)}}},
\end{aligned}
$$

(B.22)

where

$$
\begin{aligned}
& \Pi^{(i)}=P_{2}^{2(2)}\left(C_{26}^{e f f(1){ }^{(i)}} C_{62}^{e f f(1){ }^{(i)}}-C_{66}^{e f f(1){ }^{(i)}} C_{22}^{e f f(1)(i)}\right) \\
& \bar{\lambda}_{1}^{(2)}=\frac{K_{3}^{(2)} M_{2}^{(2)}-K_{2}^{(2)} M_{3}^{(2)}}{K_{1}^{(2)} M_{2}^{(2)}-K_{2}^{(2)} M_{1}^{(2)}} \\
& \bar{\lambda}_{2}^{(2)}=\frac{K_{1}^{(2)} M_{3}^{(2)}-K_{3}^{(2)} M_{1}^{(2)}}{K_{1}^{(2)} M_{2}^{(2)}-K_{2}^{(2)} M_{1}^{(2)}} \\
& \bar{\lambda}_{3}^{(2)}=\frac{\sum_{i=1}^{n} \frac{D_{2}^{(i)(2)} c^{(i)}}{D_{1}^{(i)}(1)}}{\sum_{i=1}^{n} \frac{c^{(i)}}{D_{1}^{(i)(2)}}}
\end{aligned}
$$


where

$$
\begin{aligned}
& K_{1}^{(2)}=\sum_{i=1}^{n} \frac{B_{2}^{(i)(2)} c^{(i)}}{A_{1}^{(i)(2)} B_{2}^{(i)(2)}-A_{2}^{(i)(2)} B_{1}^{(i)(2)}},
\end{aligned}
$$

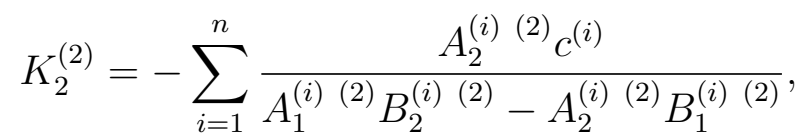

$$
\begin{aligned}
& K_{3}^{(2)}=\sum_{i=1}^{n} \frac{\left(B_{2}^{(i){ }^{(2)}} A_{3}^{(i)(2)}-A_{2}^{\left.(i){ }^{(2)} B_{3}^{(i)}{ }^{(2)}\right) c^{(i)}}\right.}{A_{1}^{(i){ }^{(2)}} B_{2}^{(i)}{ }^{(2)}-A_{2}^{(i)}{ }^{(2)} B_{1}^{(i)}(2)} \text {, } \\
& M_{1}^{(2)}=-\sum_{i=1}^{n} \frac{B_{1}^{(i)}{ }^{(2)} c^{(i)}}{A_{1}^{(i)(2)} B_{2}^{(i)(2)}-A_{2}^{(i)(2)} B_{1}^{(i)(2)}}, \\
& M_{2}^{(2)}=\sum_{i=1}^{n} \frac{A_{1}^{(i)(2)} c^{(i)}}{A_{1}^{(i)(2)} B_{2}^{(i)(2)}-A_{2}^{(i)(2)} B_{1}^{(i)(2)}}, \\
& M_{3}^{(2)}=\sum_{i=1}^{n} \frac{\left(A_{1}^{(i){ }^{(2)}} B_{3}^{(i)(2)}-B_{1}^{(i){ }^{(2)}} A_{3}^{(i)(2)}\right) c^{(i)}}{A_{1}^{(i)}{ }^{(2)} B_{2}^{(i)(2)}-A_{2}^{(i)}{ }^{(2)} B_{1}^{(i)}(2)} \text {. } \\
& \tilde{\lambda}_{1}^{(2)}=\frac{\sum_{i=1}^{n} \frac{P_{2}^{(2)} \kappa_{21}^{e f f(1)(i)} c^{(i)}}{P_{2}^{(2)} \kappa_{22}^{\text {eff }{ }^{(1)}{ }^{(i)} P_{2}^{(2)}}}}{\sum_{i=1}^{n} \frac{c^{(i)}}{P_{2}^{(2)} \kappa_{22}^{\text {eff }(1)(i)} P^{(2)}}} \\
& \tilde{\lambda}_{2}^{(2)}=\frac{\sum_{i=1}^{n} \frac{P_{2}^{(2)} \kappa_{22}^{e f f(1)(i)} c^{(i)}}{P_{2}^{(2)} \kappa_{22}^{e f f(1)(i)} P_{2}^{(2)}}}{\sum_{i=1}^{n} \frac{c^{(i)}}{P_{2}^{(2)} \kappa_{22}^{e f f(1)(i)} P^{(2)}}} \\
& \tilde{\lambda}_{3}^{(2)}=\frac{\sum_{i=1}^{n} \frac{P_{2}^{(2)} \kappa_{23}^{e f f(1)(i)} c^{(i)}}{P_{2}^{(2)} \kappa_{22}^{e f f(1)(i)} P_{2}^{(2)}}}{\sum_{i=1}^{n} \frac{c^{(i)}}{P_{2}^{(2)} \kappa_{22}^{e f f(1)}{ }^{(i)} P^{(2)}}}
\end{aligned}
$$




\section{References}

Adams, B., Bell, S., 1995. Compression strength reductions in compoiste laminates due to multiple-layer waviness. Composites Science and Technology $53(2), 207-212$.

Barmak, K., Coffey, K., 2014. Metallic films for electronic, optical and magnetic applications. Structure, processing and properties. In Series in Electronic and Optical Materials, No 40. Woodhead Publishing.

Bensoussan, A., Lions, J., Papanicolaou, G., 1978. Asymptotic methods for periodic structures. North Holland.

Briane, M., 1990. Homogénéisation de matériaux fibrés. Ph.D. thesis, Université Pierre et Marie Curie (Paris VI).

Briane, M., 1993. Three models of non periodic fibrous materials obtained by homogenization. RAIRO- Modélisation mathématique et analyse numérique 27, 759-775.

Cavalcante, M., Marques, S., Pindera, M., 2009. Transient thermomechanical analysis of a layered cylinder by the parametric finite-volume theory. Journal of Thermal Stresses 32, 112-134.

Chatzigeorgiou, G., Charalambakis, N., Murat, F., 2008. Homogenization problems of a hollow cylinder made of elastic materials with discontinuous properties. International Journal of Solids and Structures 45, 5165-5180.

Chatzigeorgiou, G., Efendiev, Y., Charalambakis, N., Lagoudas, D., 2012. Effective thermoelastic properties of composites with periodicity in cylin- 
drical coordinates. International Journal of Solids and Structures 49, 25902603.

Chatzigeorgiou, G., Efendiev, Y., Lagoudas, D. C., 2011. Homogenization of aligned "fuzzy fiber" composites. International Journal of Solids and Structures 48 (19), 2668-2680.

Chu, K.-H., 2013. Rapid transport of biomolecules along frictionless wavyrough interfaces. Open Journal of Biophysics 3, 128-132.

Grenestedt, J., Hutapea, P., 2002. Using waviness to reduce thermal warpage in printed circuits boards. Applied Plysics Letters 81(21), 4079-4081.

Guinovart-Sanjuan, D., Rodriguez-Ramos, R., Guinovart-Diaz, R., BravoCastillero, J., Sabina, F., Merodio, J., Lebon, F., Dumont, S., Conci, A., 2016. Effective properties of regular elastic laminated shell composite. Composites: Part B 87, 12-20.

Jimenez, C., Mergia, K., Lagos, M., Yialouris, P., Agote, I., Liedtke, V., Mesoloras, S., Panayiotatos, Y., Padovano, E., Badini, C., Wilhelmi, C., Barcena, J., 2016. Joining of ceramic matrix composites to high temperature ceramics for thermal protection systems. Journal of the European Ceramic Society 36(3), 443-449.

Katz, A., Trinh, C., Wright, J., Tu, W., Pindera, M., 2015. Plastic strain localization in periodic materials with wavy brick-and-mortar architectures and its effect on the homogenized response. Composites: Part B 68, 270280. 
Khatam, H., Pindera, M., 2009a. Parametric finite-volume micromechanics of periodic materials with elastoplastic phases. International Journal of Plasticity 25, 1386-1411.

Khatam, H., Pindera, M., 2009b. Thermoelastic modui of periodic multilayers with wavy architectures. Composites: Part B 40, 50-64.

Khatam, H., Pindera, M., 2010. Plasticity-triggered architectural effects in periodic multilayers with wavy microstructures. International Journal of Plasticity 26, 273-287.

Khatam, H., Pindera, M., 2012. Microstructural scale effects in the nonlinear elastic response of bio-inspired wavy multilayers undergoing finite deformation. Composites: Part B 43, 869-884.

Kugler, D., Moon, T., 2002. Identification of the most significant processing parameters on the development of fiber waviness in thin laminates. Journal of Compostie Materials 36(12), 1451-1479.

Kundalwal, S., Ray, M., 2014. Effect of carbon nanotubes waviness on the effective thermoelastic properties of a novel continuous fuzzy fiber-reinforced composite. Composites: Part B 57, 199-209.

Lakes, R., 2005. Materials with structural hierarchy. Nature 361, 511-515.

Li, D., Yang, Z., Jia, D., Wu, D., Zhu, Q., Liang, B., Wang, S., Zhou, Y., 2016. Microstructure, oxidation and thermal schock resistance of graphene reinforced sibcn ceramics. Ceramics International 42(3), 4429-4444. 
Liu, T., Yao, S.-W., Wang, L.-S., Yang, G.-J., Li, C.-X., Li, C.-J., 2016 a. Plasma-sprayed thermal barrier coatings with enhanced splat bonding for cmas and corrosion protection. Journal of Thermal Spray Technology 25(12), 213-221.

Liu, W., Lin, Z., Wang, N., Deng, Z., 2016b. Dynamic performances of thin-walled tubes with star-shaped cross section under axial impact. ThinWalled Structures 100, 25-37.

Milton, G., 2002. The Theory of Composites. Cambridge University Press.

Nie, G., Batra, R., 2010. Exact solutions and material tailoring for functionally graded hollow circular cylinders. Journal of Elasticity 99 (2), 179-201.

Popovich, V. S., Fedai, B. N., 1997. The axisymmetric problem of thermoelasticity of a multilayer thermosensitive tube. Journal of Mathematical Sciences 86 (2), 2605-2610.

Pratt, W., Allen, M., Jensen, C., 2000. Designing with wavy composite. URL http://silver.neep.wisc.edu/ msallen/DesigningSampleV2.pdf

Qu, J., Cherkaoui, M., 2006. Fundamentals of Micromechanics of Solids. John Wiley \& Sons.

Ray, M., Kundalwal, S., 2013. Effect of carbon nanotube waviness on the load transfer characteristics of short fuzzy fiber-reinforced composite. Journal of Nanomechanics and Micromechanics 10.1061/(ASCE)NM.2153$5477.0000082, \mathrm{a} 4013010$. 
Ray, M., Kundalwal, S., 2014. A thermomechanical shear lag analysis of short fuzzy fiber reinforced composite containing wavy carbon nanotubes. European Journal of Mechanics-A/Solids 44, 41-60.

Salonikitis, K., Pandremenos, J., Paralikas, J., Chryssolouris, G., 2010. Multifunctional materials: engineering applications and processing challenges. International Journal of Advanced Manufacturing Technology 49, 803-826.

Salzar, R., Pindera, M., Barton, F., 1996. Elastoplastic analysis of layered metal matrix composite cylinders - part i. Journal of Pressure Vessel Technology 118, 113-64.

Sauder, C., Brusson, A., Lamon, J., 2008. Mechanical properties of HiNicalon $\mathrm{S}$ and $\mathrm{SA} 3$ fiber reinforced $\mathrm{SiC} / \mathrm{SiC}$ mimicomposites. In Mechanical Properties and Processing of Ceramic Binary, Ternary and Composite Systems. Ceramic Engineering and Science Proceedings, Vol. 29(2). Edited by J. Salem, G. Hilmas, W. Fahrenholtz. The American Ceramic Society. Wiley.

Seidel, G., Chatzigeorgiou, G., Ren, X., Lagoudas, D., 2014. Multiscale modeling of multifunctonal fuzzy fibers based on multi-walled carbon nanotubes. Springer Series in Materials Science 188, 135-176.

Seidel, G. D., 2007. Micromechanics modeling of the multifunctional nature of carbon nanotube-polymer nanocomposites. Ph.D. thesis.

Tarn, J.-Q., Wang, Y.-M., 2001. Laminated composite tubes under extension, torsion, bending, shearing and pressuring: a state space approach. International Journal of Solids and Structures 38, 9053-9075. 
Taylor, M., Steigmann, D., 2009. Simulation of laminated thermoelastic membranes. Journal of Thermal Stresses 32(5), 448-476.

Tsalis, D., Baxevanis, T., Chatzigeorgiou, G., Charalambakis, N., 2013. Homogenization of elastoplastic composites with generalized periodicity in the microstructure. International Journal of Plasticity 51, 161-187.

Tsalis, D., Bonnay, K., Chatzigeorgiou, G., Charalambakis, N., 2015. Effective properties of multiphase composites made of elastic materials with hierarchical structure. Mathematics and Mechanics of Solids, 1-20.

Tsalis, D., Chatzigeorgiou, G., Charalambakis, N., 2012. Homogenization of structures with generalized periodicity. Composites: Part B 43, 2495-2512.

Tsukrov, I., Drach, B., 2010. Elastic deformation of composite cylinders with cylindrically orthotropic layers. International Journal of Solids and Structures $47,25-33$.

Tu, W., Pindera, M., 2013. Targeting the finite - defromation reponse of wavy biological tissues with bio - inspired material architectures. Journal of the Mechanical Behavior of Biomadical 28, 291-308. 